\title{
Moth flies (Diptera: Psychodidae) of the Moravskoslezské Beskydy Mts and the Podbeskydská pahorkatina Upland, Czech Republic
}

Jiří Kroča \& Jan Ježek

\begin{abstract}
Moth flies (Psychodidae: Diptera) of the Moravskoslezské Beskydy Mts and Podbeskydská pahorkatina Upland (Czech Republic). - Acta Mus. Siles. Sci. Natur., 64: 27-50, 2015.

Abstract: The investigation of Psychodidae is still far from finished in the NW part of the Carpathians of the Czech Republic, only 21 species have been published in scattered papers in the past. Altogether 36 genera of moth flies and 84 species including 20 threatened or rare taxa are recorded in this study from 8 localities: 5 mountainous and 3 submountainous sites. Katamormia niesiolowskii (Wagner, 1985) and Threticus negrobovi Vaillant, 1972 are new for the fauna of the Czech Republic, Katamormia strobli Ježek, 1986 and Philosepedon (Philothreticus) soljani Krek, 1971 are new for Moravia (incl. Silesia); three species are new for the Carpathians Mts generally, and 9 for the Carpathians Mts. of the Czech Republic. The total number of moth flies in CZ is increased to 175 species. The communities of moth flies of the preserved areas and localities studied here (in contrast to Kněhyňka 2.) are among the richest sites in the Czech Republic.
\end{abstract}

Key words: Psychodidae, faunistics, new records, Carpathians, Moravskoslezské Beskydy Mts, Podbeskydská pahorkatina Upland, Czech Republic

\section{Introduction}

The family Psychodidae is taxonomically one of the most difficult groups of nematocerous Diptera. Moth fly larvae are mainly semi-aquatic (a temporary part of the aquatic fauna). They develop in a wide range of specific substrates and habitats (Omelková et al. 2006, 2007, 2008; Ježek \& Omelková 2012; Ježek et al. 2013). Adults live in shaded biotopes near aquatic habitats. About 500 species of moth flies are known from Europe (Wagner 1990), 173 species had previously been documented in the Czech Republic (Ježek et al. 2012, 2013). Some taxonomic papers of Ježek (1977, 1983a,b,c, 1985, 1987a,b) have recorded 21 species of moth flies from peripheral parts of the Moravskoslezské Beskydy Mts and Podbeskydská pahorkatina Upland s. lat., including the type material of Promormia silesiensis Ježek, 1983 and Jungiella (Jungiella) hygrophila Ježek, 1987. The general characteristics of the whole area's natural history are given by Adam et al. (2003), Král (2001) and Popelár (2006). The submitted paper summarizes results of intensive inventory surveys of three "small-scale protected areas" of the Beskydy PLA in the years 2011 - 2013 (Kroča 2012, 2013a,b). It is supplemented by the results of previous research at these localities (2007-2009). Additional monitored localities from this region are not included here.

\section{Study areas and sites}

The research was conducted in three "small-scale protected areas" and adjacent streams, managed by the Beskydy PLA Administration. From a geomorphological point of view, all three areas are part of the Western Carpathians province, Outer Western Carpathians sub-province (Demek, Mackovčín et al. 2006).

Kněhyně - Čertův mlýn NNR and Salajka NNR are located in Moravskoslezské Beskydy Mts with differences in the geology and geomorphology, physicochemical parameters of water (Kroča 2012, 2013a) and types of forest vegetation (Tab 2). Two sites were monitored within the Salajka NNR (Figs 4 and 5), three sites within the Kněhyňka watercourse (incl. Kněhyně - Čertův mlýn NNR) (Figs 2 and 3).

Skalická Morávka NNM is located in the Podbeskydská pahorkatina Upland (Silesia). It is significantly different from the previously discussed "mountain small-scale protected areas" (Tab 2). The most characteristic feature is the dynamically changing Morávka River. It floods periodically, altering the main river basin as well as creating new side channels. This dynamic region is flanked by river floodplains with alluvial forests (Fig 6) and preserved cutoff stream channels (Parapotamon according to Amoros et al. 1987, Amoros \& Roux 1988). 
Three sites were observed on the cutoff stream channels (Figs 7 and 8). They represented different successional ages (based on the age and structure of the surrounding forest vegetation and condition of aquatic biotope). Another difference was the magnitude of the main channel effect on the individual sites (river flooding).

The historical localities discussed are within the sub-province of the Outer Western Carpathians, which can be defined as Western Beskids and West - Beskidian Piedmont (unit Podbeskydská pahorkatina Upland), the sites within the Outer Carpathian Depressions belong to the Northern and Western parts (only sub-unit Oderská brána; unit Moravská brána). see Tab 3.

New localities are supplemented by grid references based on Zelený (1972) and Pruner \& Míka (1996) -

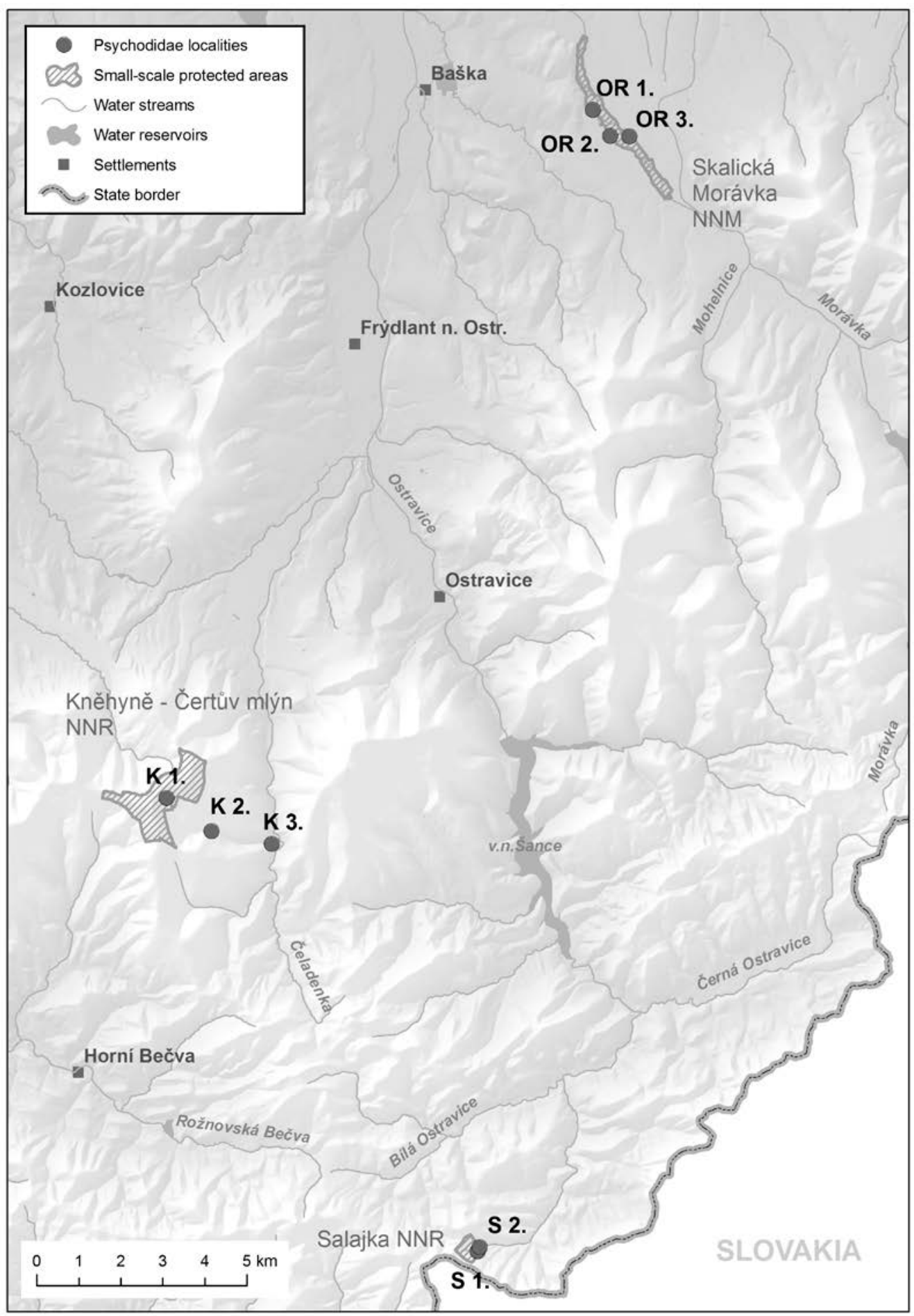

Fig 1: Map showing localities and small-scale protected areas. 


\section{Material and methods}

The data of adults presented are mainly based on inventory surveys undertaken by the first author in 2011, moreover, additional findings took place in S 2. (2007, 2008) and partially OR 1. (trapping 2009). Malaise traps (Barták's type) were installed from March / April to October / November, depending on the actual meteorological situation, and were emptied every 3-5 weeks. Captured moth flies were preserved in 70\% ethanol in the field, deposited in the laboratory and then selected specimens were mounted on slides (Canada balsam). All material J. Kroča leg. and J. Ježek det. The material is deposited in the National Museum (Natural History), Prague, Czech Republic (NMPC). All slides were numbered Inv. No. = $20972-21545$ (573 slides). The nomenclature used here mostly follows Ježek (2009a), Kvifte (2010) and Omelková \& Ježek (2012a,b,c).

The following abbrevations are used: NM - Nature Monument, NNM - National Nature Monument, NNR National Nature Reserve, NR - Nature Reserve, NP - National Park, PLA - Protected Landscape Area; K 1. Kněhyňka 1., K 2. - Kněhyňka 2., K 3. - Kněhyňka 3., OR 1., OR 2. and OR 3. - localities from NNM Skalická Morávka, S 1. - Salajka 1., S 2. - Salajka 2.; CR - critically endangered species, EN - endangered species, VU vulnerable species (categories derived from the Red List of threatened invertebrates of the Czech Republic, Ježek 2005), NS - nationally scarce (newly recorded species not assessed in the red list, however, very rare); NMPC - the collection of the Department of Entomology, National Museum in Prague, Inv. No. - Inventory Slide Number of the family Psychodidae. Abbreviations of the areas of species occurrence in the Czech Republic are shown in Tab 1.
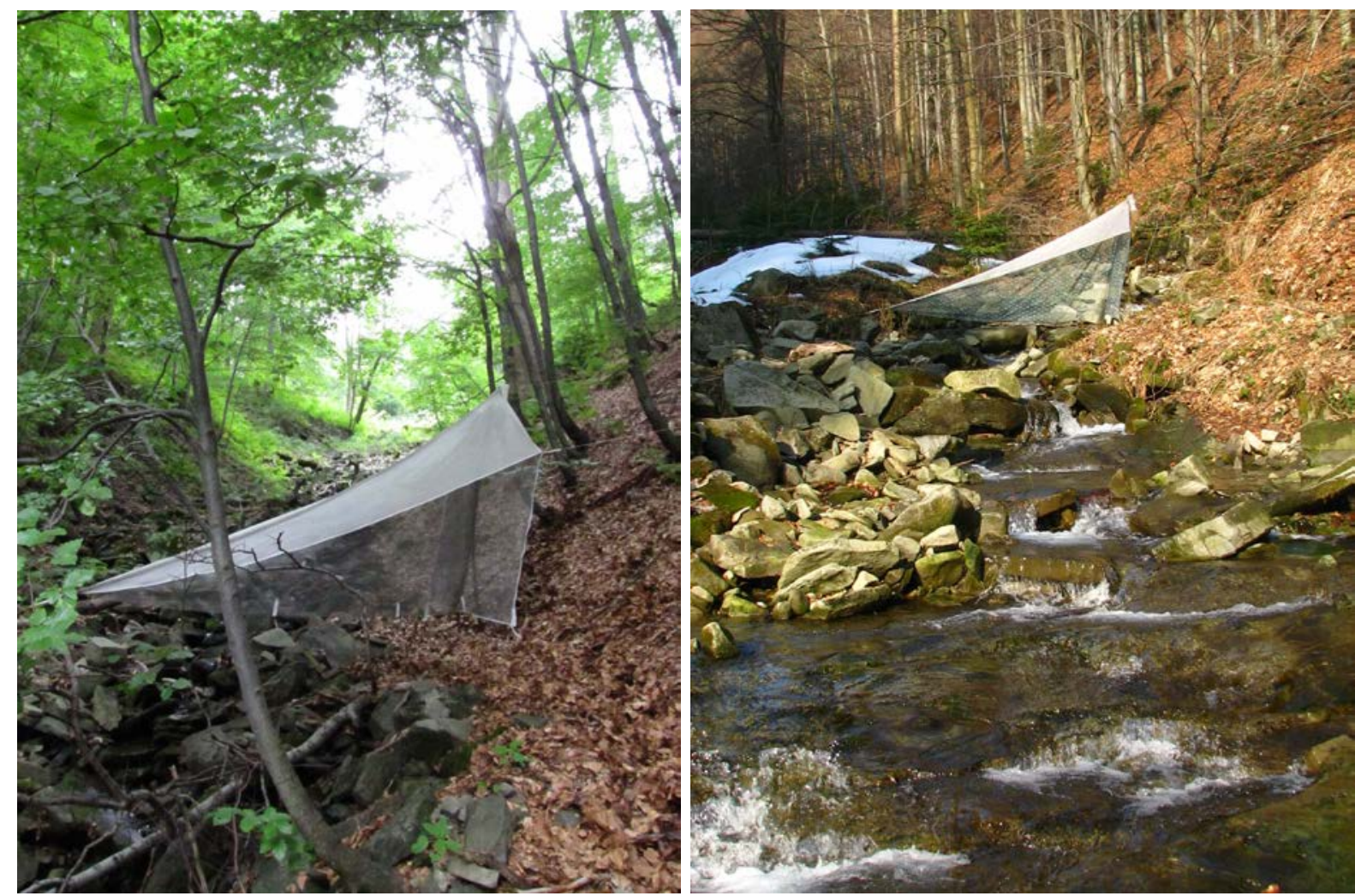

Figs 2-3: 2 - Kněhyňka 1. (Kněhyně - Čertův mlýn NNR; Beskydy PLA), photo by J. Kroča, 18 July 2011,3 Kněhyňka 2. (Beskydy PLA), photo by J. Kroča, 31 March 2011. 


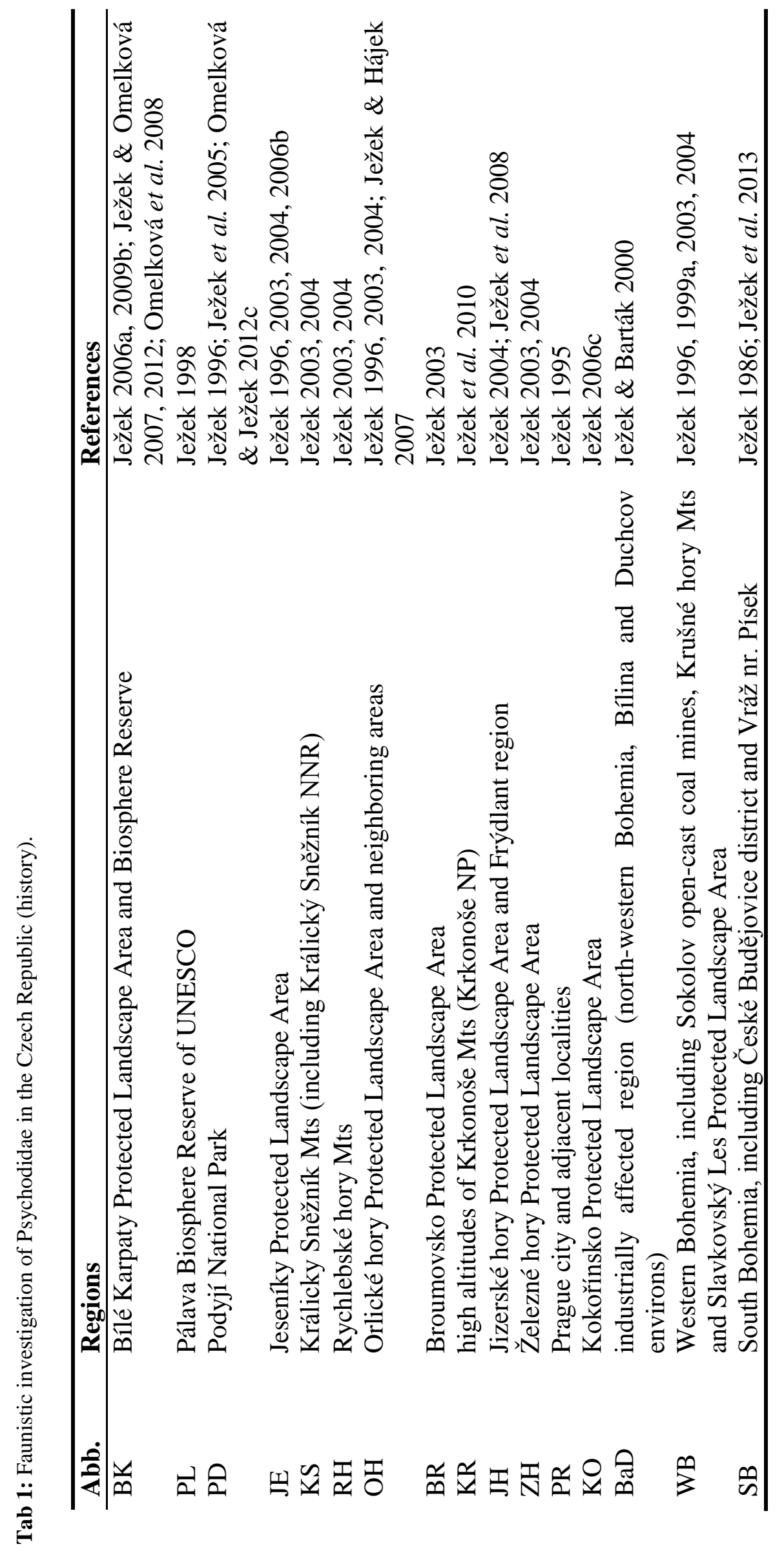




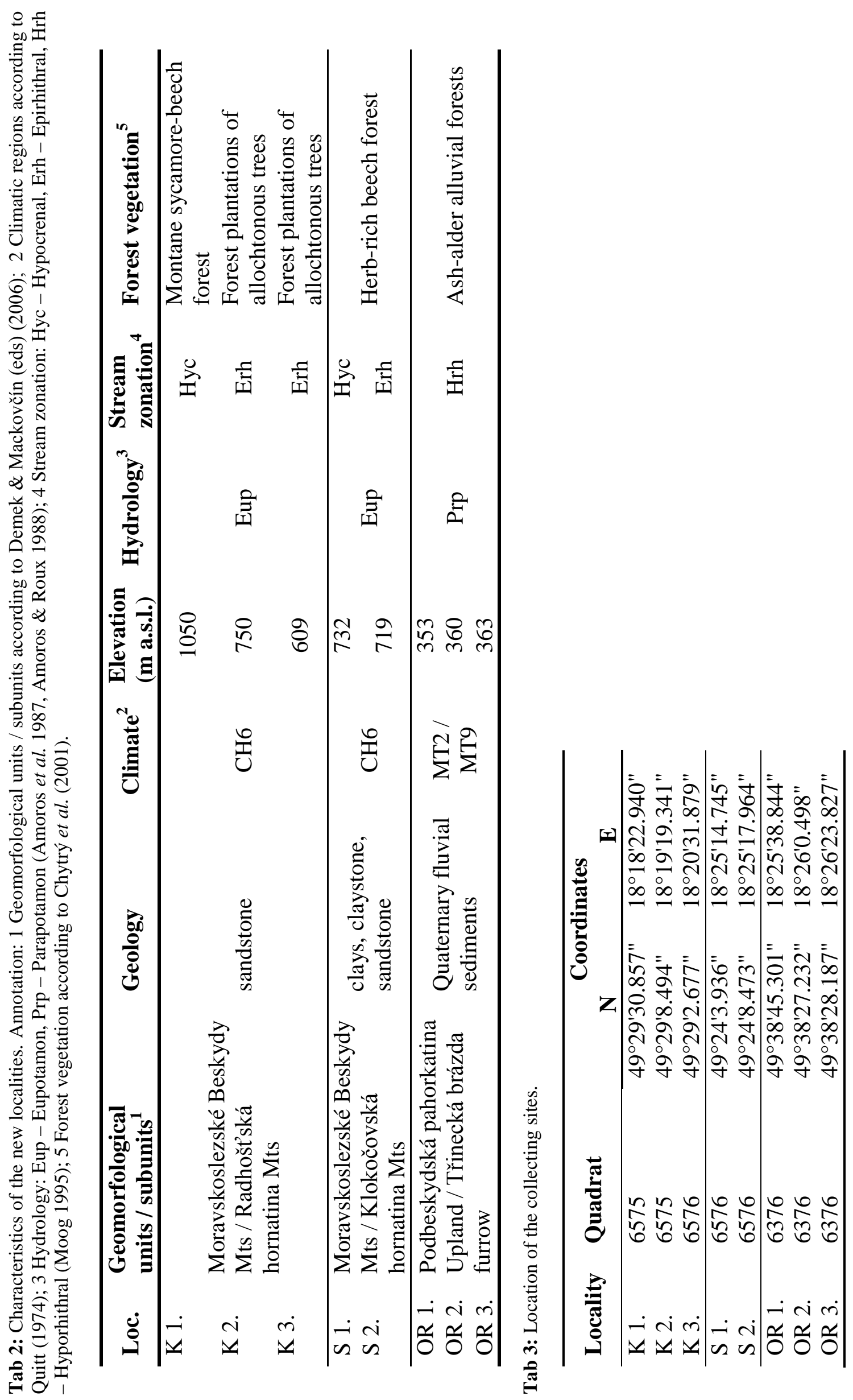




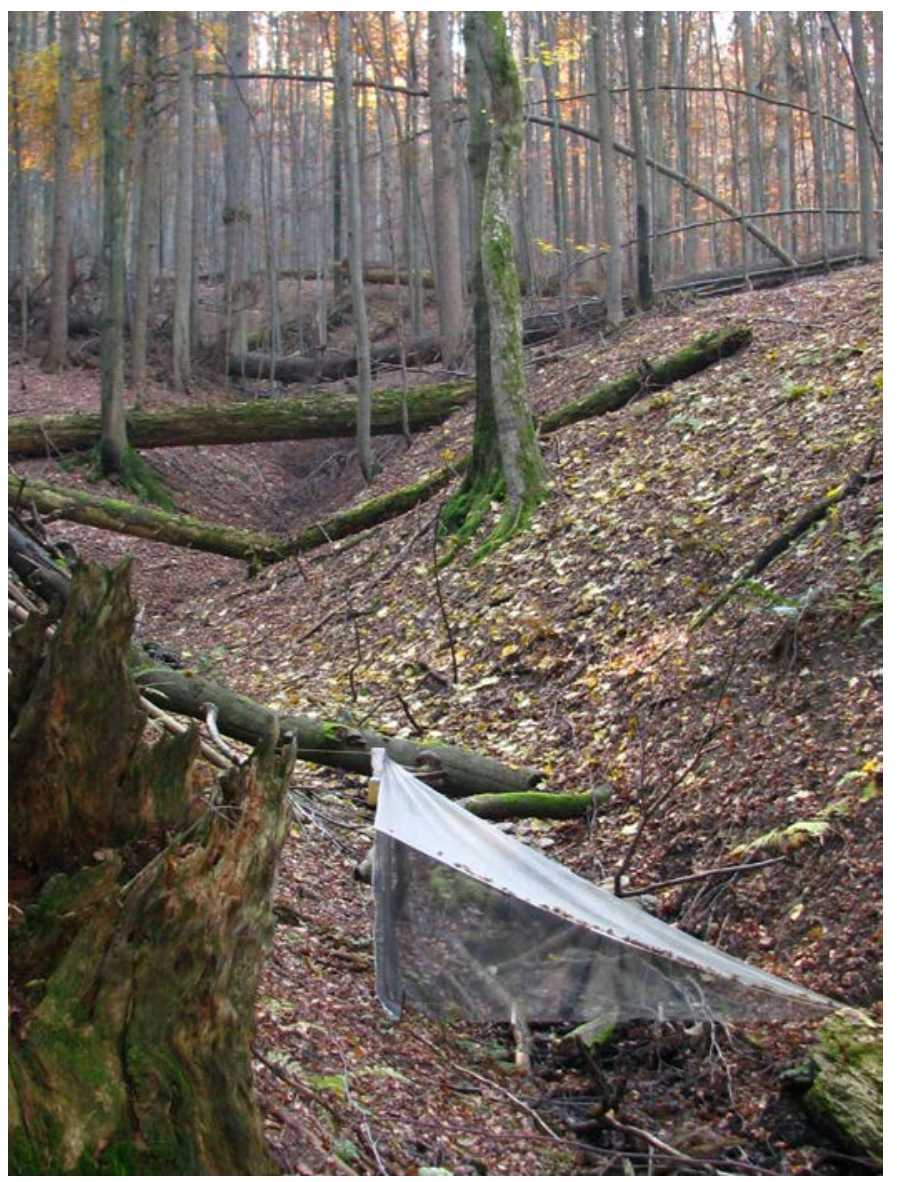

Fig 4: Locality Salajka 1. (Salajka NNR; Beskydy PLA), photo by J. Kroča, 1 November 2011.

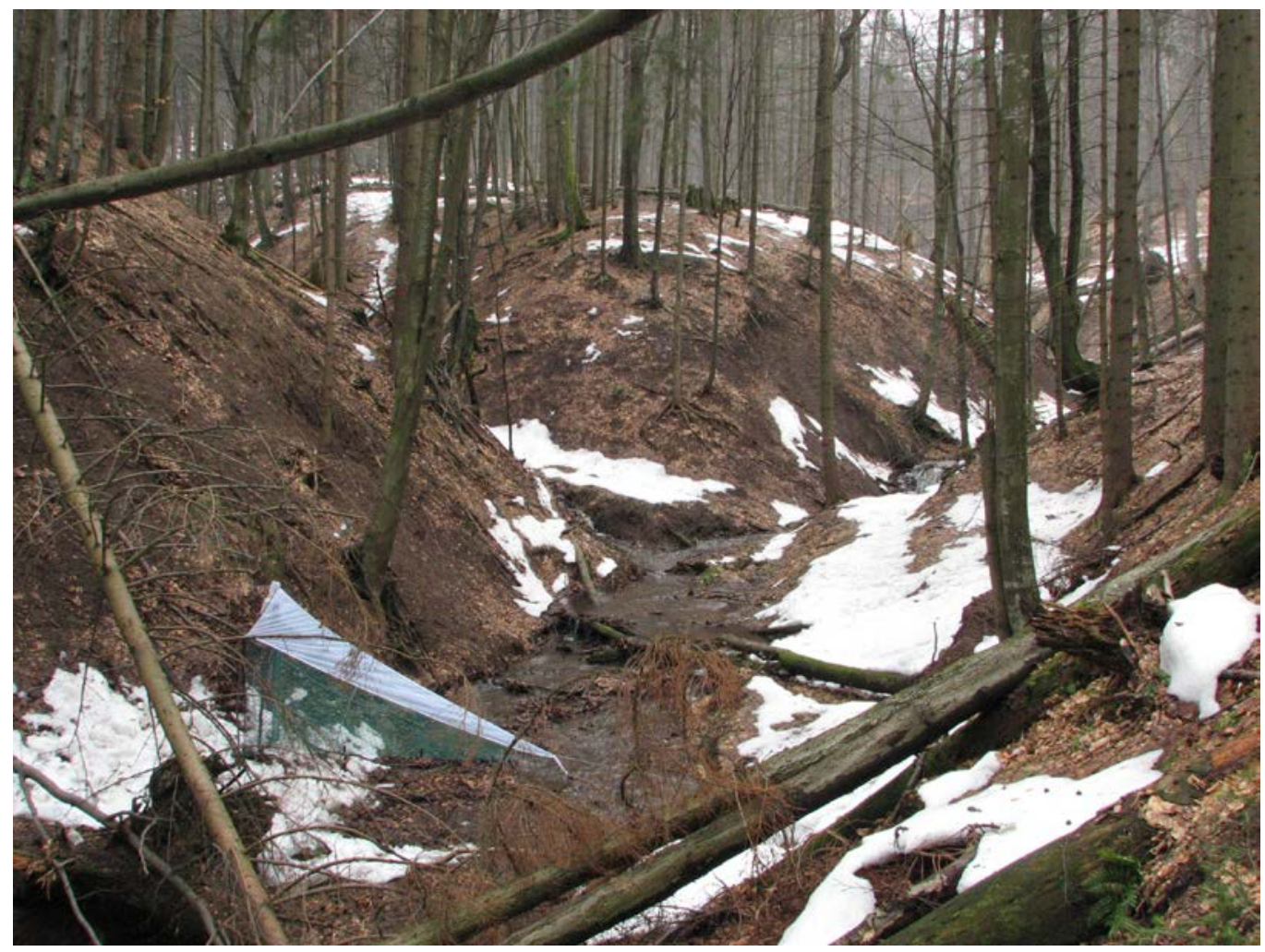

Fig 5: Locality Salajka 2. (Salajka NNR; Beskydy PLA), photo by J. Kroča, 14 March 2007. 


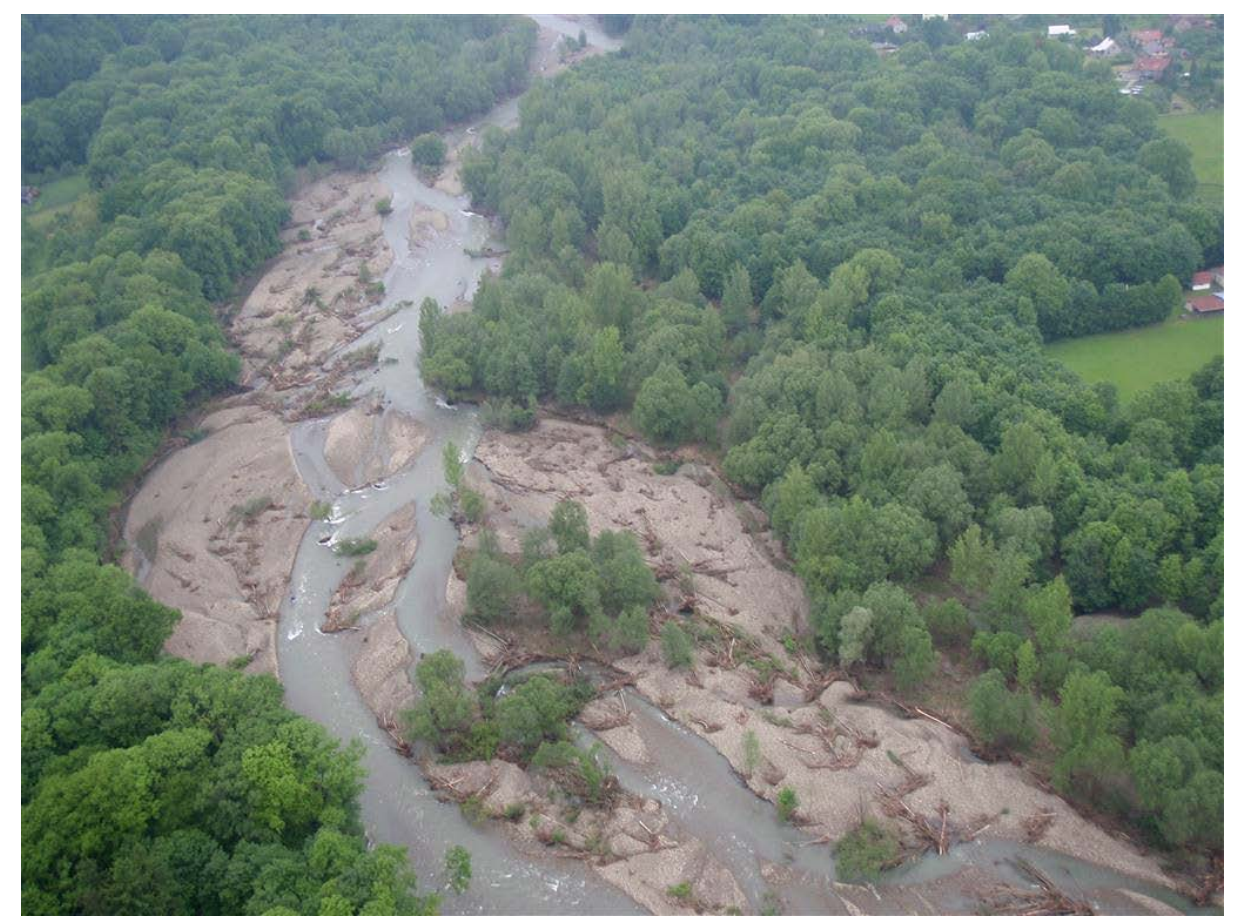

Fig 6: Skalická Morávka NNM, braided river Morávka and floodplain, situation after flood; photo by H. Ličman, 3 June 2010.

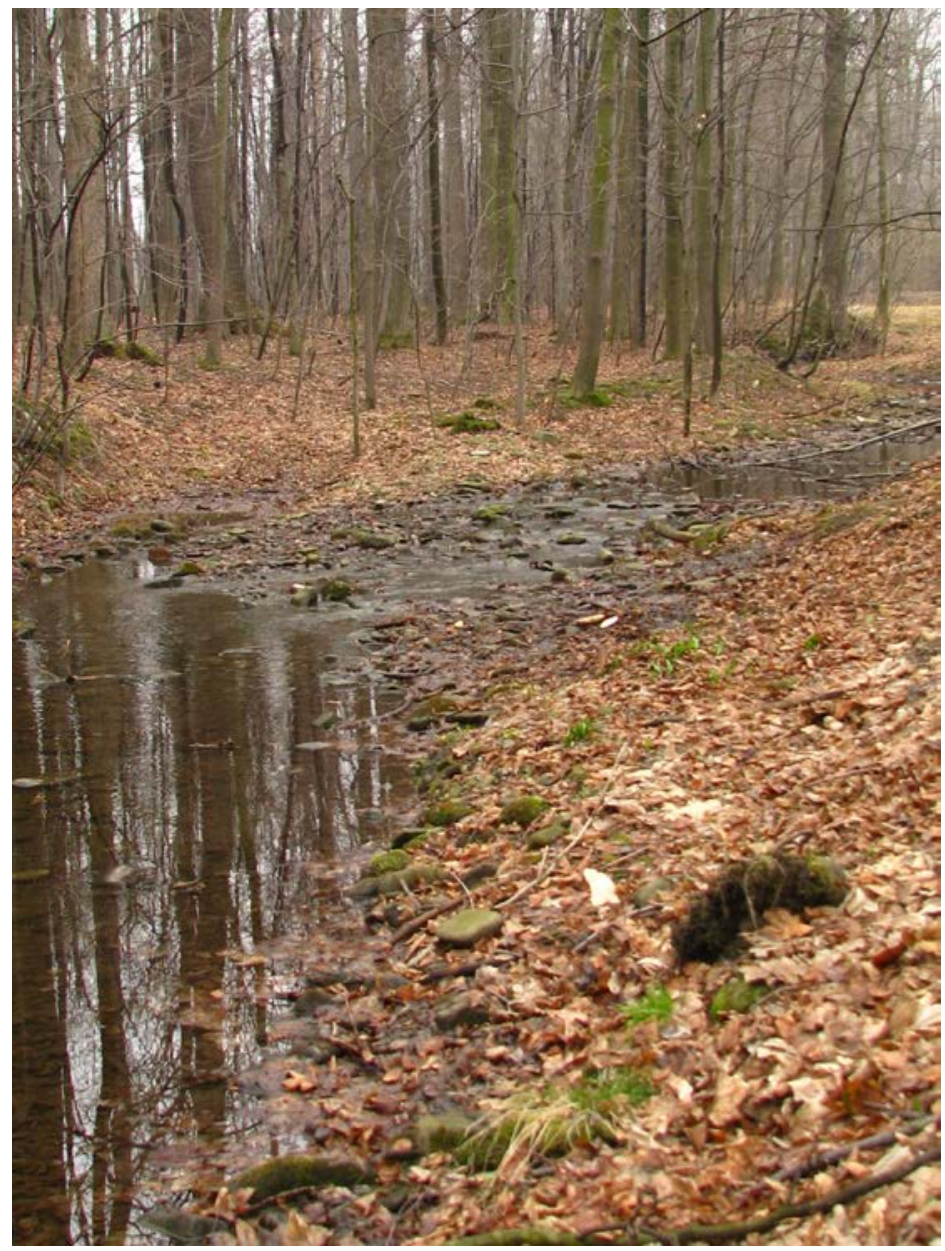

Fig 7: Locality OR 1. (Skalická Morávka NNM), photo by J. Kroča, 9 March 2013. 


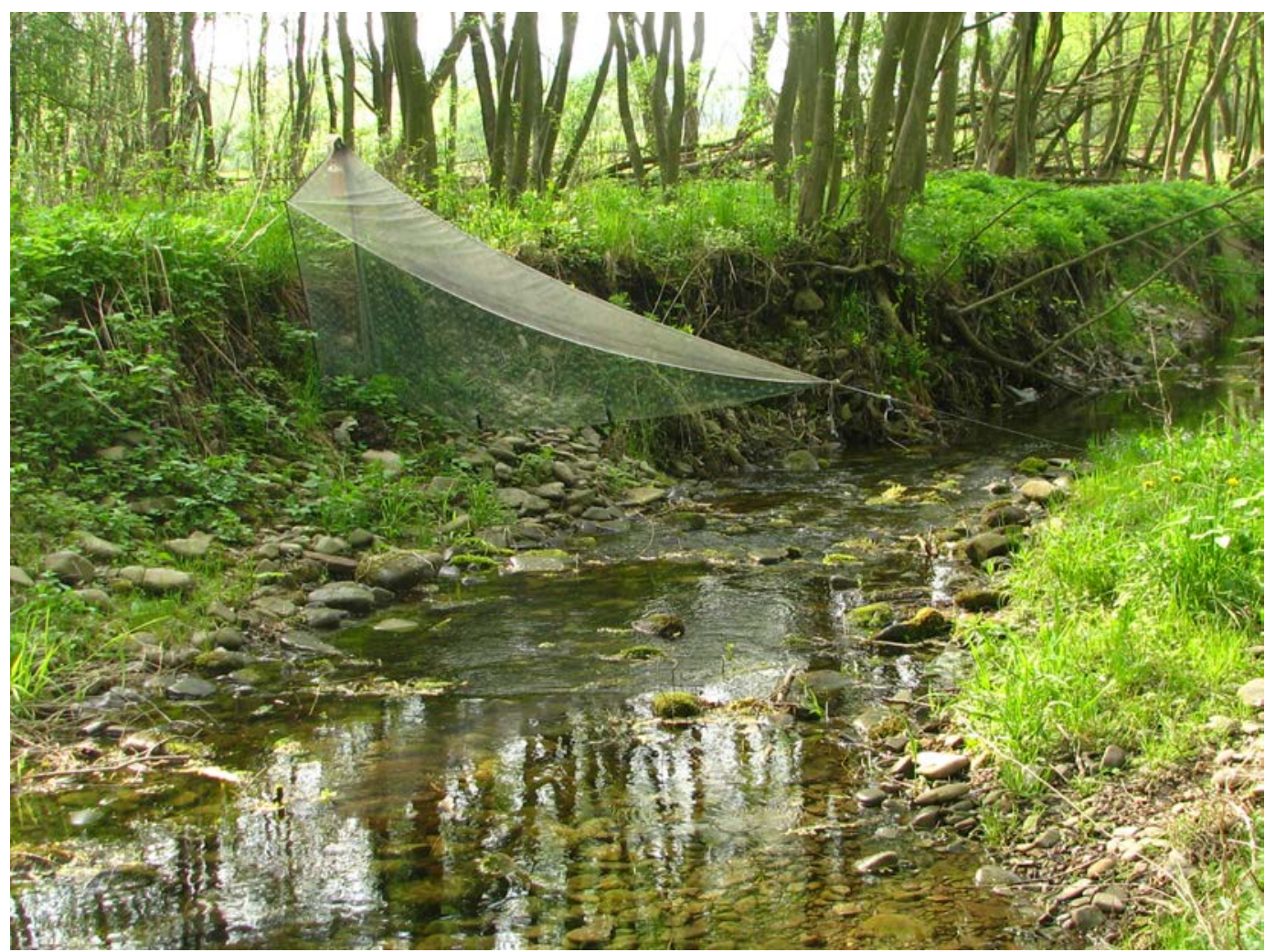

Fig 8: Locality OR 3. (Skalická Morávka NNM), photo by J. Kroča, 9 May 2013.

\section{Results}

\section{Sycorax silacea Haliday in Curtis, 1839}

Moravia: S 2.: 2. vi. - 24. vi. $2008(\overbrace{}^{\Uparrow})$.

Locally abundant species with a large area of distribution. Widespread across Central Europe, Western Europe (including the British Isles), Scandinavia, the Apennines and the Balkan Peninsula. Within the Czech Republic it was recorded in $\mathrm{BK}, \mathrm{PD}, \mathrm{JE}, \mathrm{OH}, \mathrm{JH}, \mathrm{KO}$. It inhabits a variety of forest streams, found in the moss on halfsubmerged tree branches.

\section{Katamormia niesiolowskii (Wagner, 1985) (NS)}

Silesia: SKM OR 3.: 20. v. - 20. vi. $2011(\overbrace{}^{\Uparrow})$.

A rare species, until now known from Poland and Finland (Kahanpää \& Salmela 2013, Salmela 2013). New species for the Czech Republic.

\section{Katamormia strobli Ježek, 1986 (VU)}

Silesia: SKM OR 3.: 19. vii. - 24. viii. 2011 (ठ゙).

A rare species, known only from Austria and the Czech Republic (BaD). New species for the Carpathians. New species for Moravia (including Silesia).

\section{Mormia revisenda (Eaton, 1893) (NS)}

Moravia: K 3.: 21. v. - 20. vi. 2011 (ふ).

A very rare species. Distribution: Belgium, France, Germany, Austria, Bosnia and Herzegovina, Greece and the Czech Republic (BK). It inhabits shaded slope springs and swamps, especially in beech forests. 


\section{Promormia eatoni (Tonnoir, 1940) (EN)}

Silesia: SKM OR 1.: 21. v. - 20. vi. 2011 (ð); SKM OR 2.: 30. v. - 20. vi. 2011 (ð), 20. vi. - 19. vii. 2011 (ð); SKM OR 3.: 20. v. - 20. vi. 2011 (ठ઼).

Relatively rare European species, with a large area of distribution. So far known from Bosnia and Herzegovina, Slovenia, Greece, Austria, Slovakia, Poland, Germany, Belgium and Denmark. Within the Czech Republic, $P$. eatoni was found in BK, PL, PD, JE, JH, KO and SB. The nearest records are from Ostrava - Northern Outer Carpathian depression (Ježek 1983a). Habitats: small forest streams, slope spring areas and wet pastures.

\section{Promormia silesiensis Ježek, 1983 (CR)}

Moravia: S 2.: 2. vi. - 24. vi. 2008 (ठ̋); K 3.: 21. v. - 20. vi. 2011 (ð)). Silesia: SKM OR 1.: 21. v. - 20. vi. 2011 (ð); SKM OR 2.: 30. v. - 20. vi. 2011 (ð), 20. vi. - 19. vii. 2011 (ð); SKM OR 3.: 20. v. - 20. vi. 2011 (ð).

A rare species found mainly near mountain forest streams and slope spring areas. Known from Greece, Slovakia, Poland, Slovenia, Germany and Czech Republic (BK, PD and SB). The holotype was collected in Městská Lomná env. Jablunkov (Western Beskids, Jablunkovská brázda furrow) (Ježek 1983a).

\section{Taramormia tatrica Ježek, 1984 (NS)}

Silesia: SKM OR 3.: 20. v. - 20. vi. $2011(\overbrace{}^{\Uparrow})$.

A species of meadows or shrubland spring areas, small streams and hygropetric habitats of higher altitudes. Distribution: Slovakia (Vysoké Tatry Mts) and CZ (BK).

\section{Jungiella (Jungiella) hygrophila Ježek, 1987}

Silesia: SKM OR 1.: 21. v. - 20. vi. 2011 (ठ).

A Central European species occurring in Poland and CZ (BK, PL, PD, JH, PR, KO, WB). The species was previously registered only from Polanka nad Odrou (holotypus) and Závada - Northern Outer Carpathian depressions (Ježek 1987a). Habitats: shaded spring areas and floodplains of streams. The biology is unknown.

\section{Jungiella (Psychocha) acuminata (Szabó, 1960)}

Silesia: SKM OR 1.: 21. v. - 20. vi. $2011($ ふ).

Distributed from Great Britain, France across Hungary to CZ (BK, PR). It is associated with habitats with decaying organic matter.

\section{Jungiella (Psychocha) procera Krek, 1971}

Silesia: SKM OR 1.: 21. v. - 20. vi. $2011($ (); SKM OR 2.: 30. v. - 20. vi. $2011(ð)$.

Relatively rare, little-known species, locally abundant, occurring in Bosnia and Herzegovina, Serbia and the Czech Republic (BK, PL, PR, KO). The published locality: Tichá - Podbeskydská pahorkatina Upland (Ježek 1983c). It prefers the floodplains of small streams, marshy meadows, forest spring areas, and non-forested hillside springs with tufa formations.

\section{Lepiseodina rothschildi (Eaton, 1912) (NS)}

Moravia: K 1.: 18. vii. - 23. viii. 2011 (1 §). Silesia: SKM OR 2.: 30. v. - 20. vi. 2011 (ふ).

A relatively rare European dendrolimnobiontic species, occurring from the British Isles across the countries along the North Sea coast, Italy and CZ (BK, PD, OH).

\section{Lepiseodina tristis (Meigen, 1830) (CR)}

Silesia: SKM OR 2.: 20. vi. - 19. vii. 2011 (ð); SKM OR 3.: 20. v. - 20. vi. 2011 (ð).

A rare dendrolimnobiontic species. Its distribution area ranges from Great Britain, Ireland, Belgium, the Netherlands, France, Germany, Austria, Slovakia, Lithuania, Romania to Algeria. Within the Czech Republic it was found in BK, PL, PD and SB. 


\section{Panimerus denticulatus Krek, 1971}

Silesia: SKM OR 2.: 20. vi. - 19. vii. 2011 (ð); SKM OR 3.: 20. v. - 20. vi. 2011 (ð).

Locally abundant species known from Great Britain, Ireland, Austria, Bosnia and Herzegovina, Macedonia, Greece and Czech Republic (BK, PL, PD, JE, JH, PR, KO, WB and SB). Published localities within the studied area: Podbeskydská pahorkatina Upland (Frenštát pod Radhoštěm, Rožnov pod Radhoštěm) and Northern Outer Carpathian depressions (Polanka nad Odrou, Ostrava - Poruba) (Ježek 1987b). A euryvalent species, found in different types of aquatic biotopes - shaded springs, streams, ponds, wet meadows and pastures. Common from lowlands to mountains.

\section{Panimerus notabilis (Eaton, 1893)}

Moravia: S 2.: 2. vi. - 24. vi. 2008 (ð). Silesia: SKM OR 1.: 21. v. - 20. vi. 2011 (ð).

A common species, known from the British Isles, Scandinavia, Central Europe across the Balkan Peninsula to northern Iran. Within the Czech Republic it was found in BK, PL, PD, JE, PR, BaD and SB. The nearest localities: Polanka nad Odrou and Závada in Northern Outer Carpathian depressions (Ježek 1987b). P. notabilis is associated with biotopes with decaying organic matter. It is one of the first colonizers of the early successional stages of plants (Typha) in areas of open-cast coal mines and dumps.

\section{Parajungiella ellisi (Withers, 1987) (CR)}

Silesia: SKM OR 1.: 21. v. - 20. vi. $2011\left({ }^{\Uparrow}\right)$.

A rare species known from Great Britain, Ireland, Slovakia, Austria, Russia and the Czech Republic (PD, JE, $\mathrm{OH}, \mathrm{JH}$ a WB, BaD, WB and SB). New for the Carpathians of the Czech Republic.

\section{Parajungiella longicornis (Tonnoir, 1919)}

Silesia: SKM OR 1.: 21. v. - 20. vi. 2011 (ð); SKM OR 2.: 30. v. - 20. vi. 2011 (ð)).

A very common species distributed from whole Europe to Western Siberia. In the Czech Republic is registered from $\mathrm{BK}, \mathrm{PL}, \mathrm{PD}, \mathrm{JE}, \mathrm{OH}, \mathrm{JH}, \mathrm{PR}, \mathrm{KO}, \mathrm{BaD}, \mathrm{WB}$ and SB. It prefers banks of streams (especially moss covers), ponds and forest drainages.

\section{Parajungiella serbica (Krek, 1985) (CR)}

Silesia: SKM OR 3.: 20. v. - 20. vi. $2011($ ふ).

A rare species known from Greece, Serbia, Slovakia, Poland and CZ (BK, PD, JH, BaD, WB and SB). Habitats: small streams, wetlands (inundated meadows and pastures), water reservoirs and salt marshes.

\section{Paramormia (Duckhousiella) ustulata (Walker, 1856)}

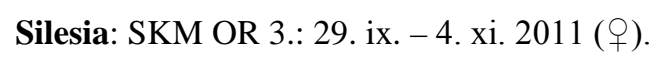

A Holarctic species, common. Its distribution area ranges from North Africa (Algeria, Morocco) and whole Europe across Asia Minor to Afghanistan and Iran, and penetrates to USA. A survey of suitable areas in CZ: PL, $\mathrm{KR}, \mathrm{JH}, \mathrm{PR}, \mathrm{BaD}, \mathrm{WB}, \mathrm{SB}$. It inhabits a vicinity of extreme localities such as salt works, thermal springs, calcareous water and mineral-rich springs.

\section{Paramormia (Paramormia) polyascoidea (Krek, 1971)}

Silesia: SKM OR 2.: 19. vii. - 22. viii. 2011 (ð̋); SKM OR 3.: 20. v. - 20. vi. 2011 (ð̋), 19. vii. - 24. viii. 2011 $\left(ठ^{1}\right)$

A European and West Siberian species ranging from Germany, Czech Republic, Poland, Austria, Bosnia and Herzegovina and Russia to the Caucasus (Abkhazia). Within the Czech Republic it was found in BK, PD, JE, $\mathrm{OH}, \mathrm{JH}, \mathrm{PR}, \mathrm{BaD}, \mathrm{WB}$ and SB. Habitats: montane swampy meadows and pastures, spring areas, lowland ponds, floodplains of meandering streams.

\section{Peripsychoda auriculata (Haliday in Curtis, 1839)}

Moravia: K 3.: 21. v. - 20. vi. 2011 (ð), 20. vi. - 18. vii. 2011 (ð). Silesia: SKM OR 1.: 21. v. - 20. vi. 2011 (ð), 19. vii. - 22. viii. 2011 (ð); SKM OR 2.: 30. v. - 20. vi. 2011 (ð), 20. vi. - 19. vii. 2011 (ð); SKM OR 3.: 20. v. - 20. vi. $2011($ ふ). 
A European and Transcaucasian species common from lowlands to highlands. Within the Czech Republic it was found in $\mathrm{BK}, \mathrm{PL}, \mathrm{PD}, \mathrm{JE}, \mathrm{OH}, \mathrm{JH}, \mathrm{PR}, \mathrm{KO}, \mathrm{BaD}, \mathrm{WB}$ and $\mathrm{SB}$. It prefers sites with decaying organic matter, e.g. ponds, silt streams, woodland pools, wet meadows and eutrophic fens.

\section{Peripsychoda fusca (Macquart, 1826) (CR)}

Moravia: S 2.: 2. vi. - 24. vi. 2008 (ठ゚).

A European species (locally abundant), with a relatively large area of distribution: British Isles and the countries along the North Sea coast, Poland and Finland form the northern boundary of occurrence; a southern line is formed by the Apennines and the Balkans. The species is rare in the Czech Republic (BK and BR). It prefers wet moss covers and leaf litter piles.

\section{Sciria advena (Eaton, 1893) (CR)}

Moravia: S 2.: 2. vi. - 24. vi. 2008 (つ); K 1.: 20. vi. - 18. vii. 2011 (つ).

A rare dendrolimnobiontic species, known from France, Germany, Great Britain, Ireland, Slovakia and the Czech Republic (BK, PL, PD, BaD, SB). It prefers primary floodplain forests.

\section{Telmatoscopus carthusianus (Vaillant, 1972)}

Moravia: S 2.: 2. vi. - 24. vi. 2008 (ठ); K 1.: 20. vi. - 18. vii. 2011 (ठ). Silesia: SKM OR 1.: 21. v. - 20. vi. 2011 (ठ); SKM OR 2.: 30. v. - 20. vi. 2011 (ठ); SKM OR 3.: 20. v. - 20. vi. 2011 (む).

Listed from France, Germany, Poland, Slovakia, Slovenia and the Czech Republic (BK, PD, JE, KR, JH, PR, $\mathrm{KO}, \mathrm{WB}, \mathrm{SB})$. It prefers forest springs, marshy meadows, streams and rivers. Common from lowlands to mountains.

\section{Telmatoscopus gressicus (Vaillant, 1972)}

Moravia: K 3.: 21. v. - 20. vi. $2011($ (ð). Silesia: SKM OR 1.: 21. v. - 20. vi. 2011 (ð); SKM OR 2.: 30 . v. - 20. vi. $2011($ ふ); SKM OR 3.: 20. v. - 20. vi. 2011 (ふ).

Locally abundant species known from France, Austria, Poland and the Czech Republic (BK, PD, JE, OH, JH, $\mathrm{PR}, \mathrm{KO}, \mathrm{BaD}, \mathrm{WB}, \mathrm{SB})$. It prefers similar localities to T. carthusianus.

\section{Feuerborniella obscura (Tonnoir, 1919)}

Moravia: S 1.: 21. v. - 21. vi. 2011 (†); S 2.: 5. viii. - 9. ix. 2008 (q).

A European species, common, penetrates from the Atlantic coast (incl. the British Isles) to Central Europe. Also known from the Apennines and the Balkan Peninsula. Within the Czech Republic it was found in BK, PL, PD, $\mathrm{JH}, \mathrm{PR}, \mathrm{BaD}, \mathrm{WB}$ and SB. The nearest published locality to our study is Ostrava - Northern Outer Carpathian depressions (Ježek 1985). It prefers wet moss cushions, springs and small streams meandering in a wide range of altitudes.

\section{Philosepedon (Philosepedon) austriacum Vaillant, 1974}

Moravia: S 1.: 21. v. - 21. vi. $2011($ đ).

Euryvalent species known from Germany, Austria, Slovakia, Slovenia, Greece, Russia and the Czech Republic (BK, PL, PD, JE, KS, OH, BaD, WB and SB). Found at a wide range of altitudes.

\section{Philosepedon (Philosepedon) hrudkai Ježek, 1999 (VU)}

Silesia: SKM OR 1.: 7. ix. - 6. x. 2009 (ð).

Very rare central European species known only from Germany and the Hercynian part of the Czech Republic (PD, JE, BaD, WB). New for the Carpathians.

\section{Philosepedon (Philothreticus) soljani Krek, 1971 (NS)}

Moravia: S 1.: 18. vii. - 23. viii. 2011 (2 §).

A very rare species, so far known only from Slovenia, Bosnia and Herzegovina, and from the Czech Republic (SB). The first record for the Carpathians. New species for Moravia (incl. Silesia). 


\section{Philosepedon (Trichosepedon) balkanicum Krek, 1971 (CR)}

Moravia: S 1.: 21. v. - 21. vi. 2011 (ठ), 18. vii. - 23. viii. 2011 (ð); S 2.: 5. ix. - 10. x. 2007 (ठ), 2. vi. - 24. vi. 2008 (ð), 5. viii. - 9. ix. 2008 (ठ), 23. ix. -21. x. 2008 (ð); K 3.: 21. v. - 20. vi. 2011 (ð).

A mountain species recorded from Germany, Slovakia, Slovenia, Bosnia and Herzegovina, it penetrates as well to Caucasus (Abkhazia). Occurrence in the Czech Republic (BK, JE, RH, OH, KR, JH, SB). In the Moravskoslezské Beskydy Mts monitored in both areas. Habitats: slope springs, avalanche tracks and near forest wells and streams.

\section{Threticus balkaneoalpinus Krek, 1972}

Moravia: S 1.: 21. v. - 21. vi. 2011 (ठ), 18. vii. - 23. viii. 2011 (ठ); S 2.: 5. ix. - 10. x. 2007 (ठ), 2. vi. - 24. vi. 2008 (ð), 5. viii. - 9. ix. 2008 (ð), 23. ix. - 21. x. 2008 (ð); K 2.: 20. vi. - 18. vii. 2011 (す); K 3.: 21. v. - 20. vi. 2011 (ð), 20. vi. - 18. vii. 2011 (ठ઼).

Known from Austria, Bosnia, Czech Republic (BK), France, Germany, Slovakia and Transcaucasus. Larvae can be found in non-forested fens, adults prefer localities near streams and rivers.

\section{Threticus negrobovi Vaillant, 1972 (NS)}

Moravia: S 1.: 21. v. - 21. vi. 2011 (ð), 18. vii. - 23. viii. 2011 (ð); K 2.: 21. v. - 20. vi. 2011 (ठ); K 3.: 21. v. - 20. vi. $2011\left(\jmath^{\lambda}\right)$.

Very rare species, so far known from Slovenia, Slovakia, Russia, Ukraine, Abkhazia and Georgia. Habitats: springs and small streams in mountainous regions. Within the Moravskoslezské Beskydy Mts. it was found in both monitored areas. New species for CZ.

\section{Threticus silvaticus Ježek, 1985 (VU)}

Moravia: K 3.: 21. v. - 20. vi. 2011 (ð).

A rare central European species, so far known only from Slovakia and Czech Republic (JE, OH, JH, WB, SB). New for the Carpathians of CZ.

\section{Trichopsychoda hirtella (Tonnoir, 1919)}

Moravia: S 2.: 5. viii. - 9. ix. 2008 (ठ̊). Silesia: SKM OR 1.: 7. ix. - 6. x. 2009 (ठ̊), 6. x. - 26. x. 2009 (ㅇ) 21. v. - 20. vi. 2011 (ð), 19. vii. - 22. viii. 2011 (ठ); SKM OR 2.: 20. vi. - 19. vii. 2011 (ठ), 19. vii. - 22. viii. 2011 (), 22. viii. - 30. ix. 2011 (ठ), 30. ix. - 4. xi. 2011 (ㅇ); SKM OR 3.: 20. v. - 20. vi. 2011 (ठ)), 19. vii. - 24. viii. 2011 (q), 29. ix. - 4. xi. 2011 (†).

A European species, common, distributed from the British Isles across Central Europe to the Apennines and the Balkan Peninsula. Reported from BK, PL, PD, JE, OH, JH, PR, KO, BaD, WB and SB in the Czech Republic, a historical locality Stonava - Northern Outer Carpathian depressions was reported by Ježek (1985). The larvae are frequent in decaying plants and fruits.

\section{Chodopsycha lobata (Tonnoir, 1940)}

Moravia: S 1.: 21. v. - 21. vi. 2011 (q), 18. vii. - 23. viii. 2011 (q), 27. ix. - 1. xi. 2011 (q); S 2.: 5. ix. - 10. x.

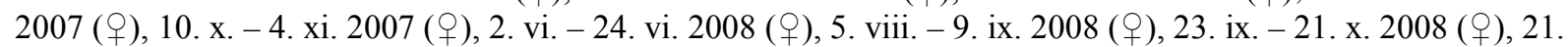
X. - 11. xi. 2008 (q); K 1.: 20. vi. - 18. vii. 2011 (q), 18. vii. - 23. viii. 2011 (q), 20. vi. - 18. vii. 2011 (q), 23. viii. - 3. x. $2011\left(\right.$ (ठ); K 3.: 20. vi. - 18. vii. $2011\left(\zeta^{\lambda}\right)$, 3. x. - 3. xi. 2011 (ठ)). Silesia: SKM OR 1.: 21. v. - 20. vi. 2011 ()); SKM OR 2.: 20. vi. - 19. vii. 2011 (q), 19. vii. - 22. viii. 2011 (q), 22. viii. - 30. ix. 2011 (q); SKM OR 3.: 20. v. - 20. vi. 2011 ().

Common widespread species ranging from Europe to Transcaucasia. Listed from BK, PD, JE, OH, KR, JH, $\mathrm{BaD}, \mathrm{WB}$ and $\mathrm{SB}$ in $\mathrm{CZ}$. The life history is associated with fungi (from lowlands to mountains).

\section{Copropsychoda brevicornis (Tonnoir, 1940)}

Silesia: SKM OR 1.: 7. ix. - 6. x. 2009 (q), 6. x. - 26. x. 2009 (†), 30. ix. - 4. xi. 2011 (q); SKM OR 3.: 20. v. - 20. vi. 2011 (ð), 19. vii. - 24. viii. 2011 (ㅇ), 29. ix. - 4. xi. 2011 ().

A Western Palaearctic saprophagous species, common, listed from Great Britain, Ireland, Denmark, Norway, Sweden, the Netherlands, Germany, France, Czech Republic (BK, PL, JE, OH), Slovakia, Russia and Turkey. 


\section{Logima albipennis (Zetterstedt, 1850)}

Moravia: S 1.: 21. v. - 21. vi. 2011 (q), 27. ix. - 1. xi. 2011 (q); S 2.: 7. iv. - 12. v. 2008 (q), 2. vi. - 24. vi. 2008 (), 5. viii. - 9. ix. 2008 (), 23. ix. - 21. x. 2008 (); K 1.: 21. v. - 20. vi. 2011 (); K 3.: 21. v. - 20. vi. 2011 (q), 20. vi. - 18. vii. 2011 (), 18. vii. - 23. viii. 2011 (q). Silesia: SKM OR 1.: 8. iv. - 22. iv. 2009 ()),

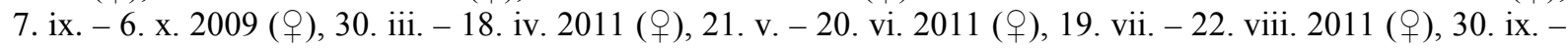
4. xi. 2011 (†); SKM OR 2.: 20. vi. - 19. vii. 2011 (†), 19. vii. - 22. viii. 2011 ()), 30. ix. - 4. xi. 2011 ()); SKM OR 3.: 20. v. - 20. vi. 2011 (ㅇ), 19. vii. - 24. viii. 2011 (ㅇ), 29. ix. - 4. xi. 2011 ()).

Very common, cosmopolitan and eurybiontic species, documented from BK, PL, PD, JE, OH, KR, JH, PR, KO, $\mathrm{BaD}, \mathrm{WB}$ and SB in CZ. There are historical localities as Western Beskids (Pržno - Vsetín district, Dolní Lomná, Jablunkov), Podbeskydská pahorkatina Upland (Tichá) and Northern Outer Carpathian depressions (Ostrava - Hrušov, Ostrava - Poruba, Stonava) (Ježek 1983b). It inhabits localities from lowlands to mountains. Larvae are saprophagous.

\section{Logima erminea (Eaton, 1893)}

Moravia: S 1.: 27. ix. - 1. xi. 2011 (ð); S 2.: 21. x. - 11. xi. 2008 (q). Silesia: SKM OR 1.: 7. ix. - 6. x. 2009 (ठ), 30. iii. - 18. iv. 2011 (), 21. v. - 20. vi. 2011 () , 19. vii. - 22. viii. 2011 (ㅇ), 30. ix. - 4. xi. 2011 (ठ); SKM OR 2.: 20. vi. - 19. vii. 2011 (†), 19. vii. - 22. viii. 2011 (q), 22. viii. - 30. ix. 2011 (†), 30. ix. - 4. xi. 2011 (†); SKM OR 3.: 20. v. - 20. vi. 2011 (ð), 19. vii. - 24. viii. 2011 (†), 29. ix. - 4. xi. 2011 (†).

A common Palaearctic species found in a wide range of altitudes, associated with fungi and caves. Within the Czech Republic it was found in BK, PL, PD, JE, OH, JH, PR, BaD, WB, SB. There is a historical locality: Louky in Northern Outer Carpathian depressions (Ježek 1983b).

\section{Logima satchelli (Quate, 1955)}

Moravia: S 1.: 19. iv. - 21. v. 2011 (q), 21. v. - 21. vi. 2011 (ð), 18. vii. - 23. viii. 2011 (q), 27. ix. - 1. xi. 2011 (ठ̂); S 2.: 10. x. - 4. xi. 2007 ()), 7. iv. - 12. v. 2008 (ㅇ), 2. vi. - 24. vi. 2008 (), 5. viii. - 9. ix. 2008 (ठ); K 1.: 18. iv. - 21. v. 2011 (), 21. v. - 20. vi. 2011 (q), 20. vi. - 18. vii. 2011 ()), 18. vii. - 23. viii. 2011

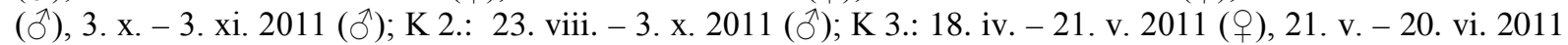
(), 20. vi. - 18. vii. 2011 (ㅇ), 18. vii. - 23. viii. $2011(§), 3$. x. - 3. xi. 2011 (ㅇ). Silesia: SKM OR 1.: 8. iv. 22. iv. 2009 (ㅇ), 7. ix. - 6. x. 2009 (ठ), 6. x. - 26. x. 2009 (ठ), 30. iii. - 18. iv. 2011 (ठ), 21. v. - 20. vi. 2011 (ð), 19. vii. - 22. viii. 2011 (ð), 30. ix. - 4. xi. 2011 (ð); SKM OR 2.: 30. v. - 20. vi. 2011 (), 20. vi. - 19. vii. 2011 (ð), 19. vii. - 22. viii. 2011 (ठ), 22. viii. - 30. ix. 2011 (), 30. ix. - 4. xi. 2011 (q); SKM OR 3.: 30. iii. 18. iv. 2011 (ठึ), 20. v. - 20. vi. 2011 (q), 19. vii. - 24. viii. 2011 (ㅇ), 29. ix. - 4. xi. 2011 (q).

A Holarctic species, eurybiontic, common, known from a wide range of altitudes. Within the Czech Republic it was listed from BK, PL, PD, JE, OH, KR, JH, PR, KO, BaD, WB, SB. Larvae are saprophagous.

\section{Logima zetterstedti Ježek, 1983}

Moravia: S 2.: 5. viii. - 9. ix. 2008 ()); K 1.: 18. iv. - 21. v. 2011 (ㅇ); K 3.: 3. x. - 3. xi. 2011 (ठ)). Silesia: SKM OR 1.: 8. iv. - 22. iv. 2009 (ふ), 30. iii. - 18. iv. 2011 (†).

A European and west Siberian species, common. Within the Czech Republic it was collected in BK, PL, PD, JE, $\mathrm{OH}, \mathrm{KR}, \mathrm{JH}, \mathrm{PR}, \mathrm{KO}, \mathrm{BaD}, \mathrm{WB}$, found in a wide range of altitudes. Historical localities: Western Beskids (Dolní Lomná, Jablůnkov) and Northern Outer Carpathian depressions (Ostrava - Hrušov, Ostrava - Poruba, Stonava, Závada) (Ježek 1983b). Larvae are saprobiontic.

\section{Psycha grisescens (Tonnoir, 1922)}

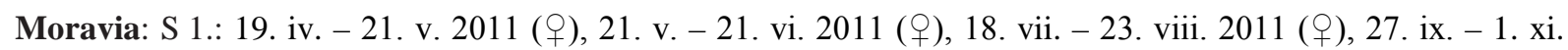
2011 (q); S 2.: 5. ix. - 10. x. 2007 (q), 10. x. - 4. xi. 2007 (ð), 7. iv. - 12. v. 2008 (q), 2. vi. - 24. vi. 2008 (†), 5. viii. - 9. ix. 2008 (q), 23. ix. - 21. x. 2008 (q), 21. x. - 11. xi. 2008 (†); K 1.: 18. iv. - 21. v. 2011 (q), 21 . v. - 20. vi. 2011 (q), 20. vi. - 18. vii. 2011 (), 18. vii. - 23. viii. 2011 (申), 3. x. - 3. xi. 2011 (申); K 2.: 18. iv. 21. v. 2011 (†), 21. v. - 20. vi. 2011 (ङ), 18. vii. - 23. viii. 2011 (q), 23. viii. - 3. x. 2011 (早), 3. x. - 3. xi. 2011 (2 §); K 3.: 18. iv. - 21. v. 2011 (§), 21. v. - 20. vi. 2011 (ㅇ), 20. vi. - 18. vii. 2011 (ㅇ), 3. x. - 3. xi. 2011 (ð). Silesia: SKM OR 1.: 8. iv. - 22. iv. 2009 (ð), 7. ix. - 6. x. 2009 (†), 6. x. - 26. x. 2009 ()), 30 . iii. 18. iv. 2011 (q), 21. v. - 20. vi. 2011 (†), 19. vii. - 22. viii. 2011 (), 30. ix. - 4. xi. 2011 (ठ)); SKM OR 2.: 20.

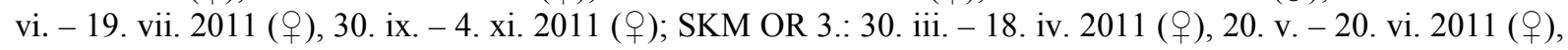
19. vii. - 24. viii. 2011 (q), 29. ix. - 4. xi. 2011 (q). 
A European species, common, occurring in the British Isles and Scandinavia across Lithuania and penetrating to central Anatolia. It was also documented in North Africa. Within the Czech Republic it was listed in BK, PL, $\mathrm{PD}, \mathrm{JE}, \mathrm{OH}, \mathrm{KR}, \mathrm{JH}, \mathrm{PR}, \mathrm{KO}, \mathrm{WB}$. Larvae are saprobiontic.

\section{Psychoda alticola Vaillant, 1973 (NS)}

Moravia: S 1.: 27. ix. - 1. xi. 2011 (ð); K 1.: 3. x. - 3. xi. 2011 (ð); K 2.: 23. viii. - 3. x. 2011 (ठ), 3. x. - 3. xi.

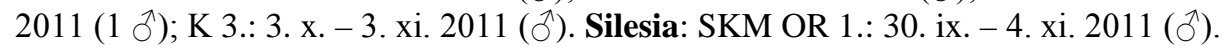

Rare species, known from Austria, France and the Czech Republic (KS, KR, JH). New for the Carpathians.

\section{Psychoda crassipennis Tonnoir, 1940 (NS)}

Silesia: SKM OR 1.: 7. ix. - 6. x. 2009 (), 30. iii. - 18. iv. 2011 (ð), 21. v. - 20. vi. 2011 (ठ), 19. vii. - 22. viii. 2011 (†); SKM OR 2.: 19. vii. - 22. viii. 2011 (†); SKM OR 3.: 20. v. - 20. vi. 2011 (す).

A European species, known from the Atlantic coast (countries incl. the British Isles) to the boreal ecoregion (Scandinavia), also reported, scarcely, from the Czech Republic (BK, OH, ZH, WB). Larvae live in the littoral zone of polluted water reservoirs, swampy areas and in sewage waters. Adults are pollinators of Arum sp.

\section{Psychoda phalaenoides (Linné, 1758)}

Moravia: S 1.: 19. iv. - 21. v. 2011 (ठ), 21. v. - 21. vi. 2011 (†), 18. vii. - 23. viii. 2011 (), 27. ix. - 1. xi. 2011 ()); S 2.: 5. ix. - 10. x. 2007 (), 10. x. - 4. xi. 2007 (ð), 7. iv. - 12. v. 2008 (ठ), 2. vi. - 24. vi. 2008 (ठ),

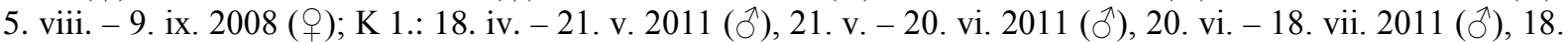
vii. - 23. viii. $2011(\overbrace{}^{\Uparrow}), 3$. x. - 3. xi. 2011 (ठ); K 2.: 18. iv. - 21. v. 2011 (ㅇ) , 21. v. - 20. vi. 2011 (ㅇ), 20. vi. 18. vii. 2011 (ð), 18. vii. - 23. viii. 2011 () , 23. viii. - 3. x. 2011 (ð); K 3.: 18. iv. - 21. v. 2011 (ㅇ), 21 . v. -

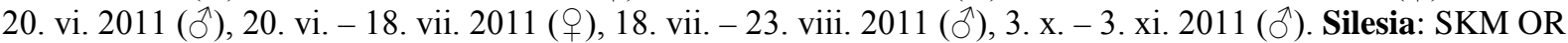
1.: 8. iv. - 22. iv. 2009 (ふ), 7. ix. - 6. x. 2009 (ふ), 6. x. - 26. x. 2009 (†), 30. iii. - 18. iv. 2011 (), 21 . v. - 20. vi. 2011 (ठ), 19. vii. - 22. viii. 2011 (†), 30. ix. - 4. xi. 2011 (ठ); SKM OR 2.: 30. v. - 20. vi. 2011 (ठ), 20. vi.

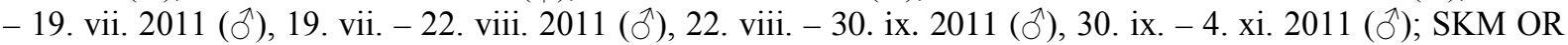
3.: 20. v. - 20. vi. 2011 (ठ), 19. vii. - 24. viii. 2011 (ठ), 29. ix. - 4. xi. 2011 (ठ).

A Holarctic polyvoltine species, common, found in a wide range of altitudes. It is consistent with documented occurrence in the Western Beskids, Podbeskydská pahorkatina Upland and Northern Outer Carpathian depressions (Ježek 1990). Within the Czech Republic it was found in BK, PL, PD, JE, OH, KR, JH, PR, KO, WB, SB. The larvae are saprobiontic.

\section{Psychoda uniformata Haseman, 1907}

Silesia: SKM OR 1.: 7. ix. - 6. x. 2009 (q), 21. v. - 20. vi. 2011 (q), 19. vii. - 22. viii. 2011 (q); SKM OR 3.:19. vii. - 24. viii. 2011 (ㅇ).

A Holarctic species. Occurrence in the Czech Republic: BK, PL, JE, OH, JH, PR, BaD, WB and SB. We are aware of only one historical locality within the Western Beskids: Bystřička (village) in Vsetínské vrchy hills (Ježek 1990a). The larvae are saprobiontic. The adults can be found in stables, cowsheds and poultry farms.

\section{Psychodocha cinerea (Banks, 1894)}

Moravia: S 1.: 21. v. - 21. vi. 2011 (), 18. vii. - 23. viii. 2011 ()), 27. ix. - 1. xi. 2011 (†); S 2.: 2. vi. - 24. vi. 2008 (q), 5. viii. - 9. ix. 2008 (q); K 3.: 21. v. - 20. vi. 2011 (ð). Silesia: SKM OR 1.: 8. iv. - 22. iv. 2009 (q), 7. ix. - 6. x. 2009 (q), 6. x. - 26. x. 2009 (q), 21. v. - 20. vi. 2011 (q), 19. vii. - 22. viii. 2011 (q), 30. ix. 4. xi. 2011 (†); SKM OR 2.: 30. v. - 20. vi. 2011 (†), 20. vi. - 19. vii. 2011 (†), 22. viii. - 30. ix. 2011 (†); SKM OR 3.: 20. v. - 20. vi. 2011 (ㅇ), 29. ix. - 4. xi. 2011 (ㅇ).

A cosmopolitan species, collected generally in a wide range of altitudes (from lowlands to mountains). Within the Czech Republic it was found in BK, PL, PD, JE, OH, JH, PR, BaD, WB, SB. The historical specimen was published from Louky - Northern Outer Carpathian depressions (Ježek 1990). The larvae are saprobiontic, associated sometimes with fungi. Adults are often observed on neglected toilets.

\section{Psychodocha gemina (Eaton, 1904)}

Moravia: S 1.: 19. iv. - 21. v. 2011 (q), 21. v. - 21. vi. 2011 (q), 18. vii. - 23. viii. 2011 (q), 27. ix. - 1. xi. 2011 (q); S 2.: 5. ix. - 10. x. 2007 (†), 2. vi. - 24. vi. 2008 (q), 5. viii. - 9. ix. 2008 (†), 23. ix. - 21. x. 2008 (†), 21. x. - 11. xi. 2008 (); K 1.: 18. iv. - 21. v. 2011 (†), 21. v. - 20. vi. 2011 (†), 20. vi. - 18. vii. 2011 (†), 18. vii. - 23. viii. 2011 (ङ); K 2.: 21. v. - 20. vi. 2011 (), 20. vi. - 18. vii. 2011 (ㅇ), 23. viii. - 3. x. 2011 ()); 


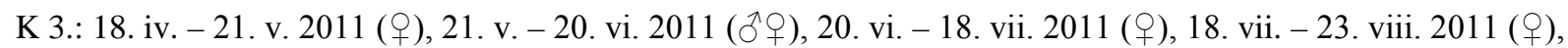
3. x. - 3. xi. 2011 (ð). Silesia: SKM OR 1.: 8. iv. - 22. iv. 2009 (†), 7. ix. - 6. x. 2009 (ð), 6. x. - 26. x. 2009

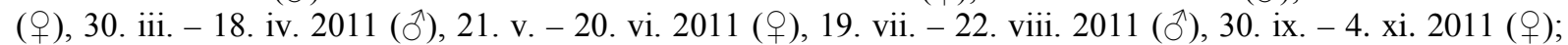
SKM OR 2.: 30. v. - 20. vi. 2011 (q), 20. vi. - 19. vii. 2011 (q), 19. vii. - 22. viii. 2011 ( + ), 22. viii. - 30. ix.

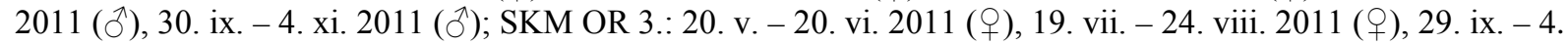
xi. $2011($ ( ) $)$.

A European species, common, documented from lowlands to mountains. Within the Czech Republic it was found in BK, PL, PD, JE, OH, KR, JH, PR, KO, BaD, WB, SB. Known from Western Beskids (Horní Lomná, Dolní Lomná, Jablunkov) (Ježek 1990). The larvae are saprobiontic, and can be collected in nests of water birds.

\section{Psychodocha itoco (Tokunaga et Komyo, 1955) (NS)}

Moravia: S 1.: 18. vii. - 23. viii. 2011 (ठ); S 2.: 5. viii. - 9. ix. 2008 (ð); K 1.: 18. iv. - 21. v. 2011 (ð), 21 . v. 20. vi. $2011($ ઊ), 18. vii. - 23. viii. $2011($ ふ).

Species published only from Japan, Finland and the Czech Republic (BK, JE, KS, OH, KR, JH, ZH). Its biology is unknown.

\section{Psychodula minuta (Banks, 1894)}

Moravia: S 1.: 19. iv. - 21. v. 2011 (q), 21. v. - 21. vi. 2011 (q), 18. vii. - 23. viii. 2011 (ठ), 27. ix. - 1. xi. 2011 (†); S 2.: 2. vi. - 24. vi. 2008 (†), 5. viii. - 9. ix. 2008 (ठ), 23. ix. - 21. x. 2008 (†); K 1.: 20. vi. - 18. vii.

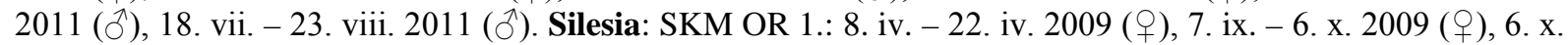
- 26. x. 2009 (早), 21. v. - 20. vi. 2011 (q), 19. vii. - 22. viii. 2011 (q), 30. ix. - 4. xi. 2011 (q); SKM OR 2.: 20. vi. - 19. vii. 2011 (†), 19. vii. - 22. viii. 2011 (q), 22. viii. - 30. ix. 2011 (q), 30. ix. - 4. xi. 2011 (す)); SKM OR 3.: 20. v. - 20. vi. 2011 (q), 19. vii. - 24. viii. 2011 (q), 29. ix. - 4. xi. 2011 (q).

A Holarctic species, common, listed from many countries, inhabiting a wide range of suitable altitudes. Within the Czech Republic it was found in BK, PL, PD, JE, OH, KR, JH, PR, BaD, WB, SB. Ježek (1990) published this species from a single Carpathian locality: Louky - Northern Outer Carpathian depressions. The larvae are saprobiontic.

\section{Psychomora mycophila (Vaillant, 1988)}

Moravia: S 2.: 10. x. - 4. xi. 2007 (ð), 5. viii. - 9. ix. $2008($ đ), 21. x. - 11. xi. 2008 (ठ); K 3.: 3. x. - 3. xi.

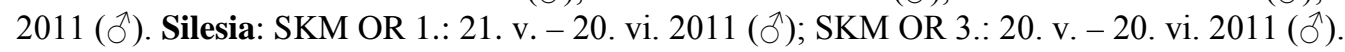

Known only from France, Switzerland, Slovakia, Slovenia and the Czech Republic (BK, PL, JH, SB). Rare, inhabiting localities in a wide range of altitudes from lowlands to mountains. The larvae are associated with fungi.

\section{Psychomora trinodulosa (Tonnoir, 1922)}

Moravia: S 1.: 19. iv. - 21. v. 2011 (†), 21. v. - 21. vi. 2011 (ð); S 2.: 5. viii. - 9. ix. 2008 (ふ); K 1.: 18. iv. -

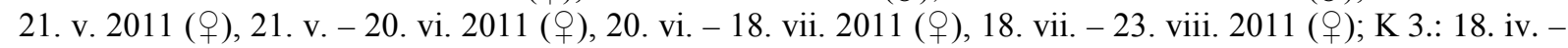
21. v. 2011 (q), 20. vi. - 18. vii. 2011 (), 18. vii. - 23. viii. 2011 (ð), 3. x. - 3. xi. 2011 (ð). Silesia: SKM OR 1.: 8. iv. - 22. iv. 2009 (ð), 7. ix. - 6. x. 2009 (†), 6. x. - 26. x. 2009 (ठ), 30. iii. - 18. iv. 2011 (†), 21. v. - 20. vi. 2011 (ð), 19. vii. - 22. viii. 2011 (ð); SKM OR 2.: 30. v. - 20. vi. 2011 (ð), 20. vi. - 19. vii. 2011 (ठ), 22. viii. - 30. ix. 2011 (ð); SKM OR 3.: 30. iii. - 18. iv. 2011 (ふ), 20. v. - 20. vi. 2011 (†), 19. vii. - 24. viii. 2011 (ふ), 29. ix. - 4. xi. $2011($ ( )

A Holarctic species, common, collected in a wide range of altitudes, known from many areas of CZ: BK, PL, PD, JE, OH, KR, JH, PR, BaD, WB, SB. Ježek (1990) documented only two localities from the Podbeskydská pahorkatina Upland and Northern Outer Carpathian depressions.

\section{Tinearia alternata (Say, 1824)}

Moravia: S 2.: 5. ix. - 10. x. 2007 (†), 5. viii. - 9. ix. 2008 (†), 23. ix. - 21. x. 2008 (†). Silesia: SKM OR 1.: 8. iv. - 22. iv. 2009 (†), 7. ix. - 6. x. 2009 (†), 6. x. - 26. x. 2009 (q), 30. iii. - 18. iv. 2011 (ठ̊), 21. v. - 20. vi. 2011 (q), 19. vii. - 22. viii. 2011 (q), 30. ix. - 4. xi. 2011 (q); SKM OR 2.: 19. vii. - 22. viii. 2011 (q), 22. viii. - 30. ix. 2011 (†), 30. ix. - 4. xi. 2011 (†); SKM OR 3.: 20. v. - 20. vi. 2011 (†), 19. vii. - 24. viii. 2011 (†), 29. ix. - 4. xi. 2011 ( $($ ) .

A cosmopolitan species, euryvalent and always very abundant in CZ: BK, PL, PD, JE, OH, KR, JH, PR, KO, $\mathrm{BaD}, \mathrm{WB}, \mathrm{SB}$. Ježek (1987b) recorded this species from only two localities in the Podbeskydská pahorkatina 
Upland (Český Těšín and Komorní Lhotka). The adults inhabit springs, streams and waterlogged meadows, larvae occur chiefly in a broad range of decaying organic materials, including faeces.

\section{Tinearia lativentris (Berdén, 1952)}

Silesia: SKM OR 3.: 20. v. - 20. vi. 2011 ()), 19. vii. - 24. viii. 2011 (ㅇ), 29. ix. - 4. xi. 2011 (ㅇ).

A Holarctic species, common, inhabits similar biotopes as T. alternata. Within the Czech Republic it was found in $\mathrm{BK}, \mathrm{PL}, \mathrm{JE}, \mathrm{OH}, \mathrm{PR}, \mathrm{BaD}, \mathrm{SB}$. The larvae are saprobiontic.

\section{Ypsydocha setigera (Tonnoir, 1922)}

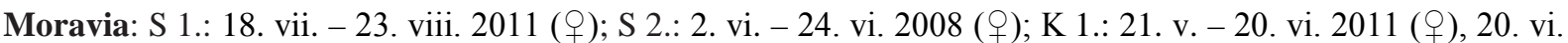
- 18. vii. 2011 (q), 18. vii. - 23. viii. 2011 (q). Silesia: SKM OR 1.: 21. v. - 20. vi. 2011 (q), 19. vii. - 22. viii. 2011 (q), 22. viii. - 30. ix. 2011 () , 30. ix. - 4. xi. 2011 ()); SKM OR 3.: 20. v. - 20. vi. 2011 ()), 19. vii. 24. viii. 2011 () .

A Holarctic species, common, documented often in CZ (BK, JE, OH, JH). The larvae are saprobiontic, collected e.g. in manure.

\section{Berdeniella manicata (Tonnoir, 1920)}

Silesia: SKM OR 1.: 19. vii. - 22. viii. 2011 (ð); SKM OR 2.: 22. viii. - 30. ix. 2011 (ð); SKM OR 3.: 20. v. 20. vi. 2011 (ठ゚), 19. vii. - 24. viii. $2011($ ふ).

A European species, locally abundant, known from the Balkan Peninsula and the Apennines, Spain, France, Belgium, Germany, Austria, Hungary, Slovakia and CZ (JE, OH, JH, WB). New for the Carpathians of the Czech Republic.

\section{Berdeniella matthesi (Jung, 1954)}

Moravia: S 1.: 21. v. - 21. vi. 2011 (ठ); K 1.: 21. v. - 20. vi. 2011 (ð); K 2.: 21. v. - 20. vi. 2011 (ठ).

A European species, locally common, listed from Germany, Austria, Italy, Slovakia and CZ (BK, JE, KS, OH, $\mathrm{KR}, \mathrm{JH}, \mathrm{ZH}, \mathrm{WB})$. It most often prefers shaded marshy habitats in hilly and montane areas.

\section{Berdeniella unispinosa (Tonnoir, 1919)}

Moravia: K 1.: 21. v. - 20. vi. 2011 (ð), 20. vi. - 18. vii. 2011 (す), 18. vii. - 23. viii. 2011 (24 ठ); K 2.: 18. vii. - 23. viii. $2011($ ( ); K 3.: 20. vi. - 18. vii. 2011 (ð), 18. vii. - 23. viii. 2011 (ð). Silesia: SKM OR 1.: 19. vii. 24. viii. $2011(\overbrace{}^{\Uparrow})$.

A European species ranging from France, the Czech Republic (boundary mountains - BK, JE, OH, KR, JH) across Slovakia to Poland. The Apennines and the Balkan Peninsula form the southern boundary of distribution. It prefers forest springs, brooks, streams and wetland habitats of avalanche tracks (higher altitudes).

\section{Berdeniella vimmeri Ježek, 1997}

Moravia: S 1.: 21. v. - 21. vi. $2011($ đ).

Probably central European species. Inhabits boundary mountains of the Czech Republic (BK, JE, OH, JH) and Slovakia. It prefers banks of forest streams, brooks and springs.

\section{Clytocerus (Boreoclytocerus) dalii (Eaton, 1893)}

Moravia: S 2.: 2. vi. - 24. vi. 2008 (ぶ); K 1.: 21. v. - 20. vi. 2011 (ぶ).

A European species, rather rare, known from Great Britain, Ireland, Denmark, Belgium, Czech Republic (BK, PD, JE, KS), Slovakia, Hungary and Slovenia. It prefers drainages of moorlands, fens and flooded meadows. Biology is unknown.

\section{Clytocerus (Boreoclytocerus) longicorniculatus Krek, 1987 (NS)}

Silesia: SKM OR 1.: 7. ix. - 6. x. 2009 (ð), 19. vii. - 22. viii. 2011 (ð).

Species known from Bosnia and Herzegovina, Czech Republic (BK, JH and SB) and Poland. The species was probably confused with $C$. ocellaris in the past, therefore, the actual distribution of longicorniculatus will be larger. Both species have the same habitat requirements. The larvae are not described. 


\section{Clytocerus (Boreoclytocerus) ocellaris (Meigen, 1804)}

Moravia: S 1.: 21. v. - 21. vi. 2011 (ð), 18. vii. - 23. viii. 2011 (ð); S 2.: 5. viii. - 9. ix. 2008 (ठ); K 3.: 18. iv. -21. v. $2011(\circlearrowleft)$. Silesia: SKM OR 1.: 7. ix. - 6. x. 2009 (ð), 19. vii. - 22. viii. 2011 (ð); SKM OR 3.: 20. v. 20. vi. $2011\left(ठ^{\Uparrow}\right)$, 19. vii. - 24. viii. 2011 (ठ゚).

A European species, common in central and western parts (including the British Isles), reaching Finland and Lithuania in the north, the Apennines and the Balkan Peninsula in the south. Within the Czech Republic it was found in BK, PL, PD, JE, OH, KR, JH, PR, KO, BaD, WB, SB. The larvae inhabit springs, small streams and a littoral zone of water reservoirs.

\section{Clytocerus (Boreoclytocerus) rivosus (Tonnoir, 1919) (CR)}

Silesia: SKM OR 2.: 20. vi. - 19. vii. $2011($ ( $)$.

An uncommon species of western and central Europe listed from the United Kingdom, Denmark, Sweden, Germany, Belgium, France, Slovakia and CZ (PL, PD, JE, OH, JH, BaD, WB). It prefers floodplain forests with oxbow rivers and backwater, hilly and mountain moors, wet meadows.

\section{Clytocerus (Boreoclytocerus) splendidus Ježek \& Hájek, 2007 (NS)}

Silesia: SKM OR 1.: 21. v. - 20. vi. 2011 (ð).

Rare species of highlands and mountains. So far recorded only from Poland and CZ (BK, PD, JE, OH, JH, ZH, WB, SB). Habitats: lakes, forest ponds, wetlands, rivers, streams, wet meadows. Biology unknown.

\section{Parabazarella subneglecta (Tonnoir, 1922)}

Moravia: S 1.: 27. ix. - 1. xi. 2011 (ð). Silesia: SKM OR 1.: 7. ix. - 6. x. 2009 (ð)); SKM OR 3.: 29. ix. - 4. xi. $2011(\overbrace{}^{\Uparrow})$.

A rare species of western and central Europe, reaching Finland and Lithuania in the north, the Balkan Peninsula and Turkey in the south. Within the Czech Republic it was found in BK, JE, OH, JH, PR, KO, WB and SB. The species prefers wet moss cushions, springs and streams.

\section{Pericoma (Pachypericoma) blandula Eaton, 1893}

Moravia: K 3.: 21. v. - 20. vi. 2011 (ð). Silesia: SKM OR 1.: 7. ix. - 6. x. 2009 (ð), 21. v. - 20. vi. 2011 (ð), 19. vii. - 22. viii. 2011 (ð); SKM OR 2.: 30. v. - 20. vi. 2011 (ð), 20. vi. - 19. vii. 2011 (ð), 19. vii. - 22. viii. 2011 (ð), 22. viii. - 30. ix. 2011 (ð); SKM OR 3.: 20. v. - 20. vi. 2011 (ð), 19. vii. - 24. viii. 2011 (す).

A European species, common, penetrates to Transcaucasia, and North Africa (Tunisia and Morocco). Within the Czech Republic it was found in BK, PD, JE, OH, JH, PR, KO, BaD, WB, SB. It prefers wet moss covers in shaded or non-shaded habitats at different altitudes.

\section{Pericoma (Pachypericoma) fallax Eaton, 1893}

Silesia: SKM OR 1.: 21. v. - 20. vi. 2011 (ð), 19. vii. - 22. viii. 2011 (ठ); SKM OR 2.: 30. v. - 20. vi. 2011

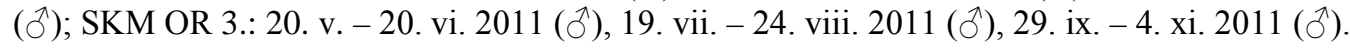

Known from Europe, western Siberia, and Caucasus (Abkhazia), more than 18 countries. Within the Czech Republic it was found in BK, PL, PD, JE, OH, PR, KO, WB, SB. A moss-dwelling species, generally common in both shaded and unshaded habitats.

\section{Pericoma (Pachypericoma) nielseni Kvifte, 2010 (NS)}

Pericoma (Pachypericoma) formosa Nielsen, 1964 - reclassified by Kvifte (2010), p. 68

Silesia: SKM OR 1.: 7. ix. - 6. x. 2009 (ठ); SKM OR 2.: 19. vii. - 22. viii. 2011 (ð).

A European species listed from Denmark, Norway, Finland, France, Slovakia and CZ (BK, PD, JH, SB). It prefers similar habitats as the previously mentioned species. 


\section{Pericoma (Pericoma) pingarestica Vaillant, 1978 (NS)}

Silesia: SKM OR 1.: 21. v. - 20. vi. 2011 (ठ).

Species documented from the Czech Republic, Slovakia, Serbia, Romania and Bulgaria. Within the Czech Republic it was found in BK, KO. It prefers hillside springs, waterlogged meadows and pastures, forest streams and wells.

\section{Pericoma (Pericoma) pseudoexquisita Tonnoir, 1940}

Silesia: SKM OR 3.: 29. ix. - 4. xi. $2011(ð)$.

Abundant European species. Within the Czech Republic it was found in JE, KR, JH, KO. New for the Carpathians of the Czech Republic.

\section{Pneumia crispi (Freeman, 1953) (EN)}

Moravia: S 1.: 18. vii. - 23. viii. 2011 (ठ); S 2.: 5. viii. - 9. ix. 2008 (ठ).

Locally abundant European species reported from Great Britain, Germany, France, Czech Republic (BK, JE, $\mathrm{OH}, \mathrm{JH}, \mathrm{BaD}$ ), Slovakia, Hungary, Romania, Bosnia and Herzegovina, Macedonia and Greece. It prefers spring areas and streams with decaying organic matter.

\section{Pneumia cubitospinosa (Jung, 1954) (EN)}

Moravia: S 1.: 21. v. - 21. vi. $2011(\hat{\jmath})$.

A European species published from Germany, Denmark, France, Switzerland, Italy, Czech Republic (BK, BR, $\mathrm{JH}$ ), Slovakia, Poland and Bosnia and Herzegovina. It inhabits wet biotopes of hilly and mountain regions.

\section{Pneumia gracilis gracilis (Eaton, 1893)}

Silesia: SKM OR 1.: 19. vii. - 22. viii. 2011 (ð); SKM OR 2.: 22. viii. - 30. ix. 2011 (ð).

A nominotypical subspecies known from eleven European countries and Caucasus. Within the Czech Republic it was found in $\mathrm{BK}, \mathrm{PD}, \mathrm{JE}, \mathrm{PR}, \mathrm{BaD}, \mathrm{WB}$ and SB. Habitats: forest slope springs, streams and wetlands. The subspecies $P$. gracilis kandavanica (Ježek, 1990) was described from Iran.

\section{Pneumia mutua (Eaton, 1893)}

Moravia: S 1.: 21. v. - 21. vi. 2011 (ð); S 2.: 2. vi. - 24. vi. 2008 (ð); K 1.: 21. v. - 20. vi. 2011 (ふ), 20. vi. 18. vii. 2011 (ठ); K 2.: 21. v. - 20. vi. 2011 (ठ).

A European species distributed from western and central countries to the northern bioregion (Scandinavia). Within the Czech Republic it was found in BK, JE, OH, KR, JH, PR, KO, WB. Habitats: moss cushions, leaf litter piles in forest streams and springs.

\section{Pneumia nubila (Meigen, 1818)}

Moravia: S 2.: 7. iv. - 12. v. 2008 (ठ); K 3.: 18. iv. - 21. v. 2011 (ð). Silesia: SKM OR 1.: 8. iv. - 22. iv. 2009

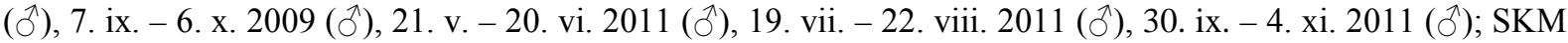
OR 2.: 30. v. - 20. vi. 2011 (ठ), 20. vi. - 19. vii. 2011 (ठ), 30. ix. - 4. xi. 2011 (ठ); SKM OR 3.: 19. vii. - 24. viii. $2011($ ふ), 29. ix. - 4. xi. $2011($ ふ).

A very common and abundant species recorded throughout Europe and the Canary Islands. Within the Czech Republic it was found in BK, PL, PD, JE, OH, JH, PR, KO, BaD, WB and SB. Habitats: shaded sites with decomposing organic matter, e.g. ponds, streams, springs, water reservoirs and swamps.

\section{Pneumia pilularia (Tonnoir, 1940)}

Moravia: S 1.: 21. v. - 21. vi. 2011 (ð); K 1.: 18. iv. - 21. v. 2011 (ð). Silesia: SKM OR 1.: 8. iv. - 22. iv. 2009

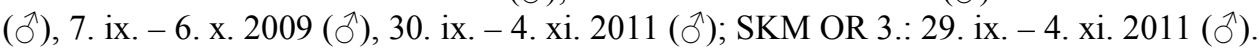

A species with a large area of distribution throughout Europe (including Spain, the British Isles and Scandinavia), North Africa (Algeria and Morocco) and Central Asia (Tajikistan). Within the Czech Republic it was found in $\mathrm{BK}, \mathrm{PL}, \mathrm{PD}, \mathrm{JE}, \mathrm{OH}, \mathrm{JH}, \mathrm{PR}, \mathrm{BaD}, \mathrm{WB}$. The larvae occur in mosses of the running water habitats (spring areas and brooks from lowlands to mountains). 


\section{Pneumia plumicornis (Tonnoir, 1922)}

Moravia: S 2.: 5. ix. - 10. x. 2007 (ठ), 10. x. - 4. xi. 2007 (ð), 23. ix. - 21. x. 2008 (ठ), 21. x. - 11. xi. 2008 $\left(ठ^{\lambda}\right)$

A European autumnal species, locally common, documented from Germany, Austria, Italy, Czech Republic (Hercynian mountains - JE, OH and JH), Slovakia and Slovenia. Habitats: small mountain streams, pools and wet meadows. New for the Carpathians of the Czech Republic.

\section{Pneumia rivularis (Berdén, 1954) (EN)}

Pericoma (Pericoma) rivularis Berdén, 1954- reclassified by Omelková \& Ježek (2012a), p. 4

Silesia: SKM OR 2.: 22. viii. - 30. ix. 2011 (ठ̋).

A rare species with a wide area of distribution from the United Kingdom to the Russian Far East and with the southern boundary limited by Germany, Czech Republic (PD, JH, ZH, BaD, WB and SB) and Slovakia. Inhabits water reservoirs, ponds, forest spring areas, swamps, wet meadows, brooks and streams. New for the Carpathians of the Czech Republic.

\section{Pneumia stammeri (Jung, 1956) (EN)}

Moravia: S 1.: 19. iv. - 21. v. 2011 (ð), 21. v. - 21. vi. 2011 (ð); S 2.: 2. vi. - 24. vi. 2008 (ð); K 1.: 21. v. 20. vi. $2011(ふ)$.

A rare European species documented from the British Isles, Scandinavian bioregion, Germany, Belgium, France, Austria, Czech Republic (BK, JE, JH and ZH), Slovakia, Hungary and Bosnia and Herzegovina. It prefers habitats with a tufa formation and the presence of mosses in wetlands near small mountain streams.

\section{Pneumia trivialis (Eaton, 1893)}

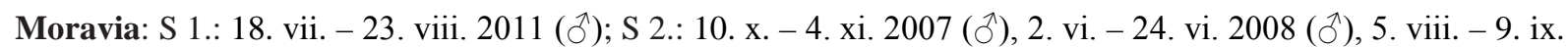
2008 (ð), 21. x. - 11. xi. 2008 (ð); K 2.: 21. v. - 20. vi. 2011 (ð); K 3.: 18. iv. - 21. v. 2011 (ð), 21. v. - 20. vi.

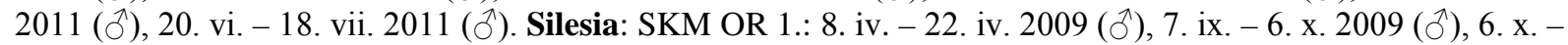
26. x. 2009 (ふ), 30. iii. - 18. iv. 2011 (つ), 21. v. - 20. vi. 2011 (ð), 19. vii. - 22. viii. 2011 (す), 30. ix. - 4. xi.

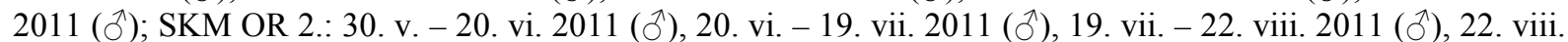
- 30. ix. 2011 (ð), 30. ix. - 4. xi. 2011 (ð); SKM OR 3.: 30. iii. - 18. iv. 2011 (ð)), 20. v. - 20. vi. 2011 (ふ), 19. vii. - 24. viii. 2011 (ð), 29. ix. - 4. xi. 2011 (ð).

A European very common species with a large distribution including many areas of its occurrence in CZ (BK, PL, PD, JE, OH, KR, JH, PR, KO, WB, SB). The larvae inhabit ponds, brooks, spring areas, reservoirs with stagnant water and swamps with decaying organic matter of shady and non-shady biotopes.

\section{Saraiella rotunda (Krek, 1970) (CR)}

Moravia: S 1.: 19. iv. - 21. v. 2011 (ठ); S 2.: 2. vi. - 24. vi. 2008 (ふ); K 2.: 23. viii. - 3. x. 2011 (ठ).

A Submediterranean mountain species, known from the Czech Republic (JE, OH, KR, JH, WB), Slovakia, Poland, Bosnia and Herzegovina, Serbia and Italy. New for the Carpathians of the Czech Republic.

\section{Szaboiella hibernica (Tonnor, 1940) (CR)}

Moravia: K 1.: 20. vi. - 18. vii. 2011 (ठ), 18. vii. - 23. viii. 2011 (2 ð) K 2.: 20. vi. - 18. vii. 2011 (ठ)), 18 . vii. - 23. viii. $2011($ ठ̊).

Widespread across Europe, known from Great Britain, Ireland, Germany, France, Spain, Italy, Czech Republic (JE, KS, RH, OH, BR, JH, ZH, WB), Slovakia, Hungary, Slovenia, Serbia, Greece and Abkhazia (Caucasus). It prefers the vicinity of mountain streams with large rocks and fallen trunks of trees, avalanche grooves, wet rocks, forest springs and wetland habitats (moors, wet meadows and pastures, swamps). New for the Carpathians of the Czech Republic.

\section{Tonnoiriella sieberti Wagner, 1993 (EN)}

Silesia: SKM OR 1.: 19. vii. - 22. viii. 2011 (ð); SKM OR 2.: 22. viii. - 30. ix. 2011 (ð), 30. ix. - 4. xi. 2011 $(\overbrace{}^{\Uparrow})$.

A rare species of higher altitudes, known from the Czech Republic (BK, PL, PD and JE), Slovakia, Poland, Macedonia, Albania and Greece. Published as well from Syria. It inhabits wetlands including waterlogged and irrigated agricultural lands, hillside springs, small streams with a significant presence of organic matter. 


\section{Ulomyia cognata (Eaton, 1893)}

Moravia: S 1.: 21. v. - 21. vi. 2011 (ठ); S 2.: 2. vi. - 24. vi. 2008 (ठ), 5. viii. - 9. ix. 2008 (ठ); K 1.: 18. iv. -

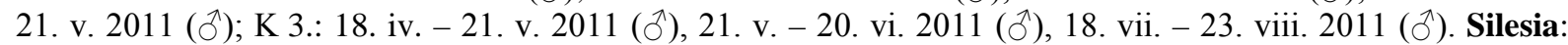
SKM OR 1.: 21. v. - 20. vi. 2011 (ठ), 19. vii. - 22. viii. 2011 (ठ).

Chiefly a European species, common from lowlands to mountains, known from Great Britain, Germany, France, Italy, Austria, Slovakia, Poland, Lithuania, Slovenia and the Czech Republic (BK, PD, JE, OH, JH, BaD, WB, SB). The larvae inhabits moss cushions in running water, hygropetric localities and decomposing leaf piles.

\section{Ulomyia fuliginosa (Meigen, 1804)}

Moravia: K 1.: 20. vi. - 18. vii. 2011 (ð); K 3.: 18. iv. - 21. v. 2011 (ठ). Silesia: SKM OR 1.: 8. iv. - 22. iv. 2009 (ð), 7. ix. - 6. x. 2009 (ð), 6. x. - 26. x. 2009 (ð), 21. v. - 20. vi. 2011 (ð); SKM OR 2.: 30. v. - 20. vi. 2011 (ð), 20. vi. - 19. vii. 2011 (ð), 19. vii. - 22. viii. 2011 (ð), 22. viii. - 30. ix. 2011 (ठ).

A widely distributed species in Europe, common from lowlands to mountains. Within the Czech Republic it was documented from BK, PL, PD, JE, OH, JH, PR, KO, BaD, WB, SB. Larvae inhabits moss cushions in flowing waters, slope springs on the banks of small streams, ponds, drainages, disused arms of rivers, swamps, marshy meadows and forest pools with decaying organic matter.

\section{Ulomyia vaseki Ježek, 2002 (EN)}

Moravia: K 1.: 20. vi. - 18. vii. 2011 (ठ), 18. vii. - 23. viii. 2011 (3 ぶ).

Rare species, listed from the Czech Republic (JE, RH, OH, JH), Slovakia (Slovenské rudohorie Mts) and Slovenia (Julijske Alpe, Kamniško-Savinjske Alpe and Karavanke). Adults prefer forest springs and small streams in mountainous areas (avalanche paths, glacial cirques). New for the Carpathians of the Czech Republic.

\section{Summary of the results and discussion}

During this study, 84 moth fly species (48 \% of the total CZ fauna) from 36 genera were recorded. The highest number of taxa was identified from Skalická Morávka NNM, with 61 species; 44 species were collected in Salajka NNR, 24 species in Kněhyně - Čertův mlýn NNR, 40 species were found in the longitudinal profile of Kněhyňka stream. The highest number of species within one site was recorded in OR 1. (45, Skalická Morávka NNM), the lowest number in K 2. (15) (Tab 4.). The diversity of the fauna of moth flies of the Moravskoslezské Beskydy Mts and Podbeskydská pahorkatina Upland is particularly high with 84 recorded species (the second most species-rich area of the Czech Republic) in comparison with the Bílé Karpaty PLA (108 species, 78 localities - Ježek \& Omelková 2012). Skalická Morávka NNM is the territory with the highest number (61 species) on the level of small-scale protected areas according to previously published papers from the Bílé Karpaty PLA - Bílé potoky NR (42), Vápenky (40), Čertoryje NNR (38), Záhumenice NM (37), Machová NR (35), Jazevčí (34) (see Ježek \& Omelková 2012). According to these results, Salajka NNR and Kněhyně - Čertův mlýn NNR (including K 2. and K 3.) are the most species-rich in the region, with the highest documented species diversity in the Carpathians of CZ. For a similar situation for individual localities see Tab 4. Altogether 20 species classified on the national Red List of threatened invertebrates (Ježek 2005) were recorded in the monitored area (10 CR, $3 \mathrm{EN}$ and $7 \mathrm{VU}), 6$ species in the NS category. It is the third richest territory in the Czech Republic: BK 26, JE 22, Moravskoslezské Beskydy Mts and Podbeskydská pahorkatina Upland 20, PD 17, OH 15. 
Tab 4: Relative species composition of the monitored areas. The percentages relate to the total number of psychodid species in CZ (175).

\begin{tabular}{lllllll}
\hline Locality & $\begin{array}{l}\text { Number of } \\
\text { species (\%) }\end{array}$ & $\begin{array}{l}\text { Number of species } \\
\text { (Red List) }\end{array}$ & CR & EN & VU & NS \\
\hline K 1. & $24(13,7)$ & 4 & 2 & 2 & 0 & 2 \\
K 2. & $15(8,6)$ & 2 & 2 & 0 & 0 & 2 \\
K 3. & $26(14,9)$ & 3 & 2 & 0 & 1 & 2 \\
\hline Kněhyně - Čertův mlýn NNR & $40(22,9)$ & 8 & 5 & 2 & 1 & 3 \\
\& Kněhyňka brook & $31(17,7)$ & 5 & 2 & 3 & 0 & 4 \\
\hline S 1. & $35(20,0)$ & 7 & 5 & 2 & 0 & 1 \\
S 2. & $44(25,1)$ & 8 & 5 & 3 & 0 & 4 \\
\hline Salajka NNR & $45(25,7)$ & 5 & 2 & 2 & 1 & 1 \\
\hline OR 1. & $36(20,6)$ & 6 & 3 & 3 & 0 & 0 \\
OR 2. & $41(23,4)$ & 5 & 3 & 1 & 1 & 2 \\
OR 3. & $61(34,9)$ & 10 & 5 & 3 & 2 & 3 \\
\hline Skalická Morávka NNM & $84(48,0)$ & 20 & 10 & 7 & 3 & 6 \\
\hline Total & & & & & \\
\hline
\end{tabular}

Katamormia niesiolowskii (Wagner, 1985) and Threticus negrobovi Vaillant, 1972 are new for the Czech Republic and increase the total number of moth flies in CZ to 175. Katamormia strobli (Ježek, 1986) and Philosepedon (Philothreticus) soljani (Krek, 1971) are new for Moravia (incl. Silesia). Katamormia strobli, Philosepedon (Philosepedon) hrudkai and Psychoda alticola are new for the Carpathians generally. Nine species - Parajungiella ellisi, Pericoma (Pericoma) pseudoexquisita, Philosepedon (Philothreticus) soljani, Pneumia plumicornis, $P$. rivularis, Saraiella rotunda, Szaboiella hibernica, Threticus silvaticus and Ulomyia vaseki are new for the Carpathians of CZ. Unfortunately, some historically documented taxa in this region by Ježek (1977, 1983a,b,c, 1985, 1987a,b) were not recorded on the monitored sites - Jungiella (Psychocha) ripicola, J. (Jungiella) soleata and $J$. (J.) valachica.

\section{Conclusion}

As already indicated, the investigation of Psychodidae in the Moravskoslezské Beskydy Mts and the Podbeskydská pahorkatina Upland is still far from finished, and after identification of the samples of all monitored additional localities, the number of species in this region will probably be significantly higher.

Acknowledgements: We express our gratitude to the management staff of the Beskydy PLA Administration for their support of our research. This study was supported by the Ministry of the Environment of the Czech Republic (MZP 0002071101) and Ministry of Culture of the Czech Republic (DKRVO 2015/13, National Museum, 0002327201). Thanks are due to Igor Konvit for processing the map and Hubert Ličman for providing photographs. We would like to thank Maxwell V. L. Barclay (London) for corrections and constructive comments on our English account.

\section{References}

Adam D., Hort L. \& Vrška T. (2003): Plán péče - NPR Salajka na období 2005 - 2014. Manuscript, depon. in: Správa CHKO Beskydy, 26 pp. (in Czech, unpublished)

Amoros C. \& Roux A. L. (1988): Interaction between water bodies within the floodplains of large rivers: function and development of connectivity. - Münster. Geogr. Arb. 29: 125-130.

Amoros C., Rostan J. C., Pautou G. \& Bravard J. P. (1987): The reversible process concept applied to the environmental management of large river systems. - Environ. Manag. 11: 607-617. 
Demek J. \& Mackovčín P. (eds) (2006): Hory a nižiny. Zeměpisný lexikon. ČR. Agentura ochrany př́rody a krajiny ČR, Brno, 582 pp. (in Czech)

Chytrý M., Kučera T. \& Kočí M. (eds) (2001): Katalog biotopů České republiky. Agentura ochrany př́rody a krajiny ČR, Praha, 304 pp. (in Czech)

Ježek J. (1977): Reinstatement of the genus Tinearia Schellenberg (Diptera, Psychodidae). - Acta Ent. Bohemoslov. 74: 232-241.

- (1983a): Contribution to the Mormini End. (Diptera, Psychodidae) in Czechoslovakia. - Acta Ent. Mus. Nat. Pragae 41: 189-212.

- (1983b): Contribution to the taxonomy of the genus Logima Eat. (Diptera, Psychodidae). - Acta Ent. Mus. Nat. Pragae 41: 213-234.

- (1983c): Contribution to the knowledge of the subgenus Psychocha Jež. of the genus Jungiella Vaill. (Diptera, Psychodidae) in Czechoslovakia. - Acta Ent. Mus. Nat. Pragae 41: 235-253.

- (1985): Contribution to the knowledge of a new subtribe Trichopsychodina (Diptera, Psychodidae) from Czechoslovakia. - Acta Mus. Nat. Pragae 40B (2): 65-92.

- (1986): Mormiini, Paramormiini a Psychodini (Diptera, Psychodidae) z okolí Českých Budějovic. Mormiini, Paramormiini and Psychodini (Diptera, Psychodidae) in České Budějovice distr. - Dipt. bohemoslov. 4: 9599. (in Czech, English summ.)

- (1987a): Jungiella hygrophila sp. n. (Diptera, Psychodidae) with rediscriptions of Czechoslovak species of Jungiella s. str. - Acta Ent. Mus. Nat. Pragae 42: 207-223.

- (1987b): Contribution to the knowledge of Panimerus Eat. (Diptera, Psychodidae) in Czechoslovakia. - Acta Ent. Mus. Nat. Pragae 42: 227-248.

- (1990): Redescriptions of nine common Palaearctic and Holarctic species of Psychodini End. Diptera: Psychodidae. - Acta Ent. Mus. Nat. Pragae 43: 33-83.

- (1995): Moth flies (Diptera, Psychodidae) inhabiting Prague city and adjacent localities with descriptions of five new species. - Acta Ent. Mus. Nat. Pragae 44: 125-165.

- (1996): New faunistic records of moth flies (Diptera, Psychodidae) from the Czech Republic. - Čas. Nár. Muz., Ř. Př́r. 165: 113-120.

- (1998): Psychodidae. In: Rozkošný R. \& Vaňhara J. (eds): Diptera of the Pálava Biosphere Reserve of UNESCO, I. - Folia Fac. Sci. Nat. Univ. Masaryk. Brun., Biol. 99: 71-77.

- (1999a): Moth flies (Diptera, Psychodidae) inhabiting wet extreme biotopes of the Sokolov open-cast coal mines and dumps, with descriptions of three new species from western Bohemia. - Sbor. Nár. Muz. v Praze, Ř. B, Př́r. V. 54(3-4) (1998): 45-60.

- (1999b): Rare and new Palaearctic Tonnoiriella - species (Diptera, Psychodidae). - Čas. Nár. Muz., ̌̌. Př́r. 168(1-4): 7-18.

- (2003): New faunistic data and check list of non Phlebotomine moth flies (Diptera, Psychodidae) from the Czech and Slovak Republics. - Čas. Nár. Muz., Ǩ. Př́r. 172: 121-132.

- (2004): New and interesting moth flies (Diptera, Psychodidae) from protected and underestimated natural areas of the Czech Republic. - Čas. Nár. Muz., Ǩ. Př́r. 173 (1-4): 113-128.

- (2005): Psychodidae (koutulovití). Pp. 259-261. In: Farkač J., Král D. \& Škorpík M. (eds): Červený seznam ohrožených druhů České republiky. Bezobratlí. Red list of threatened species in the Czech Republic. Invertebrates. Agentura ochrany př́rody a krajiny ČR, Praha, 758 pp.

- (2006a): Faunistic records, Psychodidae. In: Kinkorová J. (ed.): Dipterologica Bohemoslovaca Vol. 13. Acta Univ. Carol., Biol. 50: 139-141.

- (2006b): Psychodidae (Diptera) of the Jeseníky Protected Landscape Area and its environs with descriptions of two new Berdeniella species from the Czech Republic. - Acta Ent. Mus. Nat. Pragae 46: 151-192.

- (2006c): Moth flies (Psychodidae, Diptera) of Kokoř́nsko Protected Landscape Area.- Bohemia Centr. (Praha) 27: 423-429.

- (2009a): Psychodidae Newman, 1834. In: Jedlička L., Kúdela M. \& Stloukalová V. (eds): Checklist of Diptera of the Czech Republic and Slovakia. Electronic version 2. http://zoology.fns.uniba.sk/diptera2009. Accessed 31.9.2013.

- (2009b): Further new records of moth flies (Insecta, Diptera, Psychodidae) from the Czech Republic and Slovakia. - Folia Faun. Slov. 14: 101-105.

Ježek J. \& Barták M. (2000): Psychodidae. In: Barták M. \& Vaňhara J. (eds): Diptera in an industrially affected region (north-western Bohemia, Bílina and Duchcov environs). I. - Folia Fac. Sci. Nat. Univ. Masaryk. Brun., Biol. 104: 93-100.

Ježek J., Barták M. \& Vaněk J. (2010): Koutulovití (Diptera, Psychodidae) vysokých poloh Krkonoš. Psychodidae (Diptera) of the high altitudes of Krkonoše Mts. - Opera Corcon. 47: 265-274.

Ježek J. \& Hájek J. (2007): Psychodidae (Diptera) of the Orlické hory Protected Landscape Area and neighbouring areas with descriptions of two new species from the Czech Republic. - Acta Ent. Mus. Nat. Pragae 47: 237-285. 
Ježek J., Kubík Š. \& Barták M. (2005): Psychodidae. Pp. 78-85. In: Barták M. \& Kubík Š. (eds): Diptera of Podyjí National Park and its environs. Česká zemědělská univerzita v Praze, Fakulta agrobiologie, potravinových a přírodních zdrojů, PowerPrint, Praha, 432 pp.

- (2013): Psychodidae (Diptera) of Vráž nr. Písek (Czech Republic), 189-198 pp. In: Kubík Š. \& Barták M. (eds), Workshop on biodiversity, Jevany, Česká zemědělská univerzita v Praze, 436 pp.

Ježek J., Lukáš J., Kvifte G.M. \& Oboňa J. (2012): New faunistic records of non-biting moth flies (Diptera: Psychodidae) from the Czech Republic and Slovakia. - Klapalekiana 48: 121-126.

Ježek J. \& Omelková M. (2007): Faunistic records from Czech Republic and Slovakia: Diptera, Psychodidae. - Acta Zool. Univ. Comen. (Bratislava) 47: 250-253.

- (2012): Moth flies (Diptera: Psychodidae). In: Malenovský I., Kment P. \& Konvička O. (eds): Species inventories of selected arthropod groups in the Bílé Karpaty Protected Landscape Area and Biosphere Reserve. - Acta Mus. Morav., Sci. Biol. 96(2) 2011: 763-802.

Ježek J., Vonička P. \& Preisler J. (2008): Koutulovití (Diptera: Psychodidae) Jizerských hor a Frýdlantska. Psychodidae (Diptera) of the Jizerské hory Mts and Frýdlant region (northern Bohemia, Czech Republic). Sbor. Severočes. Muz., Přrír. V. (Liberec) 26: 129-151.

Kahanpää J. \& Salmela J. (eds) (2013): Draft Catalogue of Finnish Flies (Insecta: Diptera). www.elisanet.fi/jere.kahanpaa/diptera/list/FIdiptera.pdf

Král V. (2001): Fyzická geografie Evropy. Academia, Praha, 348 pp. (in Czech)

Kroča J. (2012) (Ms.): Inventarizační průzkum NPR Salajka z oboru hydrobiologie. Závěrečná zpráva, depon. in: Správa CHKO Beskydy, Rožnov pod Radhoštěm; Výzkumný Ústav Vodohospodářský T.G.M., v.v.i., pobočka Brno, 28 pp. (in Czech, unpublished)

- (2013a) (Ms.): Inventarizační průzkum NPR Kněhyně - Čertův mlýn z oboru hydrobiologie. Závěrečná zpráva, depon. in: Správa CHKO Beskydy, Rožnov pod Radhoštěm; Výzkumný Ústav Vodohospodářský T.G.M., v.v.i., pobočka Brno, 32 pp. (in Czech, unpublished)

- (2013b) (Ms.): Inventarizační průzkum NPP Skalická Morávka z oboru hydrobiologie. Závěrečná zpráva, depon. in: Správa CHKO Beskydy, Rožnov pod Radhoštěm; Výzkumný Ústav Vodohospodářský T.G.M., v.v.i., pobočka Brno, 104 pp. (in Czech, unpublished)

Kvifte G.M. (2010): Pericoma nielseni nom. nov., a replacement name for Pericoma formosa Nielsen, 1964, preoccupied by Pericoma formosa Meunier, 1905 (Diptera: Psychodidae). - Zootaxa 2726: 68.

Moog O. (ed.), Bauernfeind E., Berger H., Blatterer H., Car M., Contreras-Lichtenberg R., Eder E., Foissner W., Graf W., Grasser U., Hödl W., Hörner K., Janecek B., Jäch M., Kodada J., Kohmann F., Mohrig W., Moritz C., Nesemann H., Orendt C., Pöckl M., Reischütz P.L., Saxl R., Schödl S., Šporka F., Waringer J., Weichselbaumer P., Weinzierl A., Weibmair W., Wöss E., Zettel H. (1995): Fauna Aquatica Austriaca - Comprehensive Species Inventory of Austrian Aquatic Organisms with Ecological Data. First Edition, Wasserwirtschaftskataster, Bundesministerium für Land- und Forwirtschaft, Wien.

Omelková M., Bojková J., Rozkošný R., Horsák M. \& Helešic J. (2006): Nové a zajímavé larvy dvoukřídlých (Diptera) na flyšových prameništích. P. 61. In: Sborník př́íspěvků 14. konference ČLS a SLS. Česká limnologická společnost, Nečtiny, 167 pp. (in Czech)

- (2007): The Diptera taxocoenoses in then Western Carpathian spring fens: preliminary results. P. 234. In: SEFS-5 Symposium for European freshwater sciences programme and abstracts. EFFS - European Federation for Freshwater Sciences, Palermo, Italy, 288 pp.

Omelková M. \& Ježek J. (2012a): Two new species of Pneumia Enderlein (Diptera, Psychodidae, Psychodinae) from the Palaearctic region. - Zootaxa 3180: 1-18.

- (2012b): Two new species of Philosepedon Eaton (Diptera, Psychodidae, Psychodinae) from Europe, with comments on subgeneric classification. - Zootaxa 3275: 29-42.

- (2012c): A new species of the genus Trichomyia (Diptera: Psychodidae) and new faunistic data on nonphlebotomine moth flies from the Podyjí NP and its surroundings (Czech Republic). - Acta Ent. Mus. Nat. Pragae 52(2): 505-533.

Omelková M., Ježek J., Starý J., Roháček J. \& Holinka J. (2008): Dvoukřídlí (Diptera). True flies (Diptera). Pp. 294-298 and 304-305. In: Jongepierová I. (ed.): Louky Bílých Karpat. Grasslands of the White Carpathian Mountains. ZO ČSOP Bílé Karpaty, Veselí nad Moravou, 461 pp.

Popelář P. (ed.) (2006): Plán péče o Národní přírodní rezervaci Kněhyně - Čertův mlýn 2007-2016. Manuscript, depon. in: Správa CHKO Beskydy, 34 pp. (in Czech, unpublished)

Pruner L. \& Míka P. (1996): Seznam obcí a jejich částí v České republice s čísly mapových polí pro sítové mapování fauny. List of settlements in the Czech Republic with associated map field codes for faunistic grid mapping system. - Klapalekiana 32 (Suppl.): 1-175.

Quitt E. (1974): Klimatické oblasti ČSR - mapa 1:1 000 000. Kartografie n. p. Praha. (in Czech)

Salmela J. (2013): List of Finnish "semiaquatic flies". https://sites.google.com/site/cranefliesoffinland/list-offinnish-craneflies 
Wagner R. (1990): Family Psychodidae. Pp. 11-65. In: Soós A. Á. \& Papp L. (eds): Catalogue of Palaearctic Diptera, Vol. 2, Psychodidae - Chironomidae. Akadémiai Kiadó, Budapest, 499 pp.

Zelený J. (1972): Entwurf einer Gliederung der Tschechoslowakei für Zwecke der faunistischen Forschung (mit 5 Abb.). - Zpr. Českoslov. Spol. Ent. při ČSAV 8: 3-16.

Authors’ addresses: Jiří Kroča, T. G. Masaryk Water Research Institute, p.r.i., Department of Water Quality Protection, Mojmírovo nám. 16, CZ - 61200 Brno, Czech Republic

E-mail: jiri_kroca@vuv.cz

Jan Ježek, Department of Entomology, National Museum, Cirkusová 1740, CZ -193 00 Praha 9 - Horní Počernice, Czech Republic

E-mail: jan.jezek@quick.cz 